\title{
Directional Wave Spectra Observed during JONSWAP 1973
}

\author{
D. E. HasselmanN \\ Meteorologisches Institut, Universität Hamburg, Germany \\ M. DUNCKEL \\ Max-Planck-Institut für Meteorologie, Hamburg, Germany \\ J. A. EwING \\ Institute of Oceanographic Sciences, Wormley, Surrey, England \\ (Manuscript received 25 September 1979, in final form 17 March 1980)
}

\begin{abstract}
Estimates of the directional wave spectrum obtained from the meteorological buoy of the University of Hamburg and a pitch-and-roll buoy of the Institute of Oceanographic Sciences are reported from a series of measurements made within the framework of the Joint North Sea Wave Project during September 1973.

Three main aspects were considered. First, the properties and parameterization of the directional spectrum were studied when the waves were generated by steady winds without any significant swell contribution. The results do not support the parameterization proposed by Mitsuyasu et al. (1975) and are in agreement with a parameterization in which the peak frequency is the relevant scale parameter. Second, comparisons are made between two independent methods of fitting the data exactly by means of a maximum likelihood technique (Long and Hasselmann, 1979) and a least-squares technique. The two methods give very similar fits to the observed data. Finally, the response of the directional wave spectrum to veering winds is considered and a simple model is constructed as a first attempt to describe some of the observations.
\end{abstract}

\section{Introduction}

Although there have been many studies of the development and characteristics of the frequency spectrum of wind waves, there are comparatively few systematic studies of the directional wave spectrum apart from the recent work of Mitsuyasu et al. (1975), Tyler et al. (1974), Regier and Davis (1977) and Forristall et al. (1978), and the early investigations of Coté et al. (1960), Longuet-Higgins et al. (1963) and Ewing (1969).

One of the main aims of this study was to investigate the properties of the directional spectrum and, in particular, the parameterization proposed by Mitsuyasu et al. (1975) applicable to steady wind conditions using data collected from two kinds of system during the Joint North Sea Wave Project. The principle of measurements of the directional wave spectrum for the pitch-and-roll buoy is well known (Longuet-Higgins et al. , 1963); the method of obtaining directional information from a meteorological buoy is based on the measurement of all three components of acceleration and rotation and, in principle, gives identical information to that obtained from the pitch-and-roll buoy. Details are explained in Appendix A.
Under conditions of veering winds the directional information is difficult to interpret and methods for constructing the directional distribution which use all the information available are helpful. The recently proposed maximum likelihood technique (Long and Hasselmann, 1979) was used and the results were compared with an improved parametric fit similar to that discussed by Cartwright and Smith (1964).

\section{Measurements}

The measurements were made during September 1973 at the JONSWAP site (Hasselmann et al ., 1973; Günther et al., 1979). The pitch-and-roll buoy was deployed from a ship located at station 10 approximately $52 \mathrm{~km}$ off the island of Sylt in a water depth of $22 \mathrm{~m}$. The meteorological buoy was deployed from a ship at station 8 (water depth of $18 \mathrm{~m}$ ) about $27 \mathrm{~km}$ offshore. Measurements were made at intervals of either 2 or $4 \mathrm{~h}$ at station 10, while at station 8 the data were recorded continuously and later analyzed at approximately 1 and $2 \mathrm{~h}$ intervals. The times of measurements are shown in Fig. 1 which also displays the wind speed $U$ (at a level of $10 \mathrm{~m}$ ) 

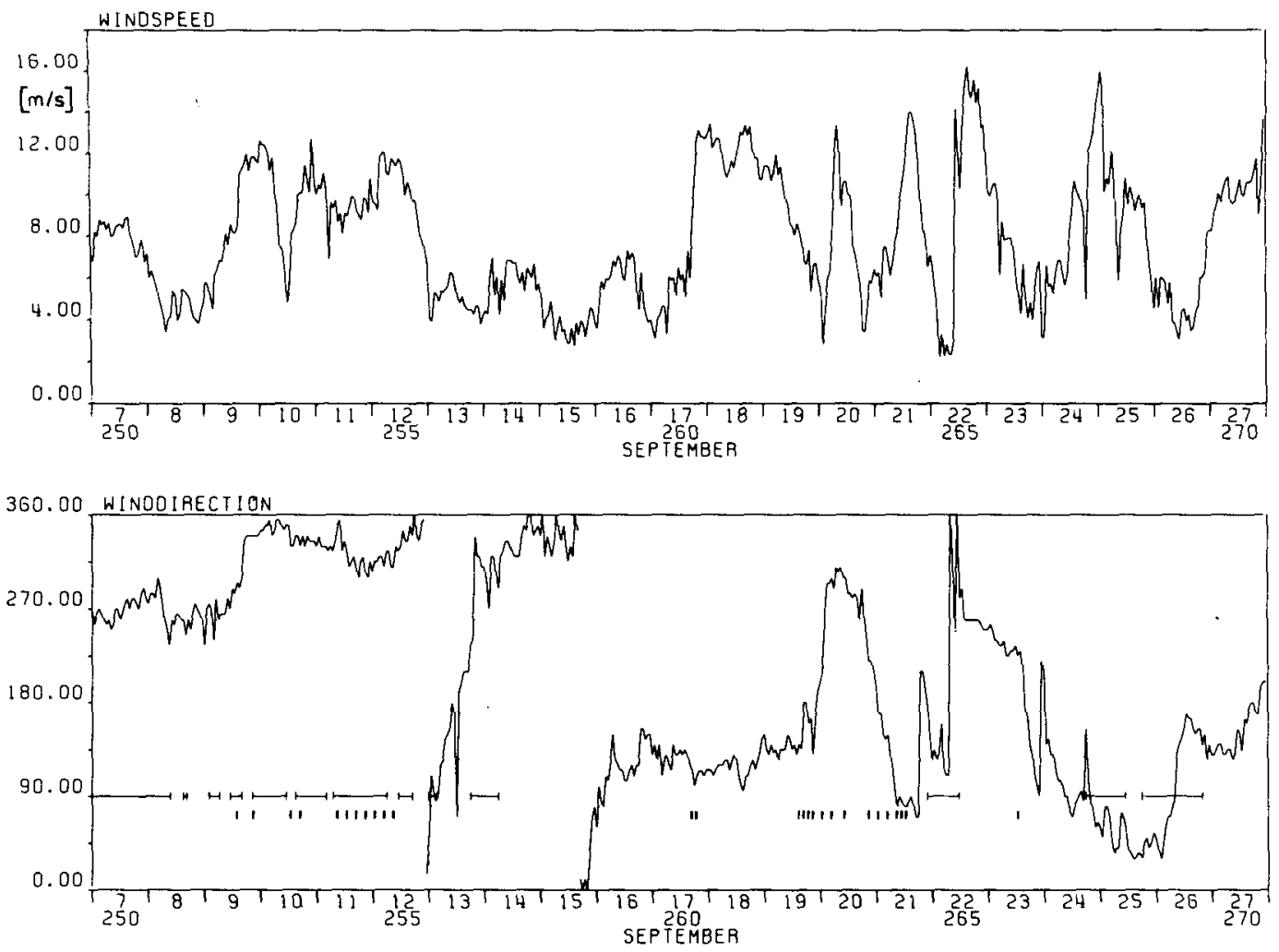

FIG. 1. Wind speed and direction from where the wind is blowing $\left(\delta_{w}+180^{\circ}\right)$ during JONSWAP 73 (10 min averages). Also shown are the times of measurement at station 8 and the half-hourly intervals at station 10 (short blocks).

and direction measured at station 8 discussed in Brümmer et al. (1974).

Fig. 1 shows that well-defined steady meteorological conditions were never encountered during September 1973. Nevertheless, a number of cases (Table 1) could be selected for the first analysis of directional properties under fairly steady wind conditions in the absence of swell. Criteria for the selection of these runs (data set A) were 1) $c_{m} \leqslant U$, where $c_{m}$ is the phase velocity at the spectral peak; 2 ) the absence of swell; and 3) the steadiness of the wind field with regard to speed and direction and judged subjectively from Fig. 1. The analysis of veering wind cases is discussed in Section 6.

\section{Data analysis}

Cross-spectral estimates were computed using fast Fourier transform techniques. Each record of length equal to $32 \mathrm{~min}$ was divided into 15 non-overlapping segments of length $128 \mathrm{~s}$ and cross spectra were formed by averaging over all segments. The sampling interval was $0.5 \mathrm{~s}$. This procedure gives spectral estimates with a resolution of $1 / 128 \mathrm{~Hz}$ and 30 degrees of freedom. The buoy diameters of $2.1 \mathrm{~m}$ (meteorological buoy) and $1.2 \mathrm{~m}$ (pitch-and-roll buoy) mean that information can be obtained up to at least $0.5 \mathrm{~Hz}$. At frequencies $<9 / 128 \mathrm{~Hz}$ the meteorological buoy has problems with low-frequency noise, while the pitch-and-roll buoy gives results down to about $5 / 128 \mathrm{~Hz}$. For this reason we have analyzed results from both buoys over the range $9 / 128 \mathrm{~Hz}$ to $0.5 \mathrm{~Hz}$. We also found it necessary to reduce the amount of information by averaging directional spectra over five adjacent frequencies thus giving estimates with 150 degrees of freedom.

Strong currents can have an important influence on the estimation of directional spectra (Forristall et al., 1978). In our situation tidal currents of $\sim 0.4 \mathrm{~m} \mathrm{~s}^{-1}$ have negligible influence over the frequency range considered.

Using the methods of Longuet-Higgins et al. (1963), we thus have estimates from both buoys of the Fourier coefficients $a_{1}, b_{1}, a_{2}, b_{2}$ of the spreading function $S(f, \theta)$ and the frequency spectrum $E(f)$ :

where

$$
E(f, \theta)=E(f) S(f, \theta),
$$

and

$$
\int_{0}^{2 \pi} S(f, \theta) d \theta=1
$$

$S(f, \theta)=\pi^{-1}\left\{1 / 2+\sum_{n=1}^{\infty} a_{n} \cos n \theta+b_{n} \sin n \theta\right\}$ 
Table 1. Data set A.

\begin{tabular}{|c|c|c|c|c|c|c|c|c|c|}
\hline Day number/time & $\begin{array}{l}\text { Sta- } \\
\text { tion }\end{array}$ & $\begin{array}{c}U^{\mathrm{a}} \\
\left(\mathrm{m} \mathrm{s}^{-1}\right)\end{array}$ & $\delta_{w}{ }^{\mathbf{a}}$ & $\begin{array}{c}H_{s}^{\mathrm{a}, \mathrm{b}} \\
(\mathrm{m})\end{array}$ & $\begin{array}{l}f_{m}{ }^{\mathrm{a}} \\
(\mathrm{Hz})\end{array}$ & $U / c_{m}{ }^{\mathrm{a}}$ & $s_{\max }{ }^{\mathrm{c}}$ & $f_{s}^{\mathrm{c}}$ & $N_{\text {run }}{ }^{d}$ \\
\hline $2500400-2500600$ & 8 & $8.5-8.7$ & $76-83$ & $0.64-0.72$ & $0.23-0.24$ & $1.31-1.34$ & 7.2 & 0.273 & 3 \\
\hline $2500700-2501600$ & 8 & $8.0-8.9$ & $74-100$ & $0.75-0.88$ & $0.19-0.22$ & $1.08-1.17$ & 7.5 & 0.234 & 9 \\
\hline $252 \quad 1200-252 \quad 1400$ & 8 & $8.1-8.6$ & $110-112$ & $0.66-0.71$ & $0.20-0.24$ & $1.05-1.34$ & 10.3 & 0.195 & 2 \\
\hline $252 \quad 1600-2522000$ & 8 & $10.3-11.5$ & $112-160$ & $0.85-0.84$ & $0.15-0.20$ & $1.15-1.35$ & 10.4 & 0.195 & 2 \\
\hline $2522200-2530630$ & 8 & $11.5-12.5$ & $160-166$ & $1.74-1.88$ & $0.14-0.15$ & $1.03-1.11$ & 13.5 & 0.195 & $2^{\mathrm{e}}$ \\
\hline $2550300-2551500$ & 8 & $9.4-12.0$ & $140-165$ & $1.22-1.65$ & $0.14-0.17$ & $1.03-1.31$ & 11.9 & 0.234 & 9 \\
\hline $2672100-2680200$ & 8 & $12.9-15.0$ & $240-260$ & $0.92-1.39$ & $0.17-0.21$ & $1.68-1.78$ & 14.3 & 0.195 & $4^{f}$ \\
\hline $2680300-2680700$ & 8. & $10.1-11.2$ & $219-260$ & $1.12-1.33$ & $0.17-0.19$ & $1.16-1.31$ & 18.1 & 0.195 & $4^{f}$ \\
\hline $2681800-2682000$ & 8 & $9.6-9.8$ & $212-230$ & $0.82-0.92$ & $0.20-0.21$ & $1.26-1.33$ & 13.1 & 0.195 & $3^{\mathrm{r}}$ \\
\hline $252 \quad 1300-252 \quad 2010$ & 10 & $8.2-11.4$ & $110-160$ & $0.79-1.80$ & $0.14-0.20$ & $1.02-1.04$ & 11.2 & 0.156 & 2 \\
\hline 2550420 & 10 & 11.9 & 136 & 1.68 & 0.14 & 1.09 & 6.1 & 0.195 & 1 \\
\hline 2601620 & 10 & 6.8 & 300 & 0.55 & 0.25 & 1.10 & 4.9 & 0.273 & 1 \\
\hline $262 \quad 1400-262 \quad 1630$ & 10 & $7.5-8.2$ & $314-330$ & $0.88-0.89$ & $0.20-0.21$ & $1.01-1.06$ & 8.5 & 0.195 & 2 \\
\hline
\end{tabular}

a Ranges given do not indicate a development in time.

${ }^{\text {b }} H_{s}=4\left\langle\zeta^{2}\right\rangle^{1 / 2}$.

c Maximum observed volume of $s$, found at frequency $f_{s}(\mathrm{~Hz})$.

d Number of runs.

"At intermediate times runs with $U / c_{m} \leqslant 0.9$.

' Wind slowly turning, these runs are also part of data set B. Waves not completely aligned with the wind, $\delta_{1}-\delta_{w} \approx 40^{\circ}$ at the spectral peak.

and we count directions clockwise from north. $\mathrm{Al}-$ ternatively, we may introduce $r_{1}, r_{2}, \delta_{1}$ and $\delta_{2}$ by writing (3.2) as

$$
S(f, \theta)=(2 \pi)^{-1}\left\{1+2 \sum r_{n} \cos n\left(\theta-\delta_{n}\right)\right\} .
$$

The rms spread angle $\theta_{2}$ is obtained as

$$
\theta_{2}=\left(2-2 r_{1}\right)^{1 / 2} \text {. }
$$

\section{Conventional analysis of data set $A$}

We may fit the parameters $r_{1}$ and $\delta_{1}$ of (3.3) with a directional distribution

where

$$
S_{1}(f, \theta)=M\left(\theta, s_{1}, \delta_{1}\right),
$$

$$
\begin{gathered}
M(\theta, p, \alpha)=\frac{1}{N(p)} \cos ^{2 p}\left(\frac{\theta-\alpha}{2}\right), \\
s_{1}=\frac{r_{1}}{1-r_{1}} .
\end{gathered}
$$

For convenience we use the notation $1 \mathrm{M}$ to describe a fit consisting of one function like (4.2). Later we consider a combination of two such functions and denote this by $2 \mathrm{M}$.

For data set $\mathrm{A}, \delta_{1}$ and $\delta_{2}$ were nearly always closely aligned with the direction toward which the wind was blowing, $\delta_{w}$. The exceptions are listed in Table 1. These exceptions, nevertheless, were clearly generation cases, as could be seen from inspection of the one- and two-dimensional spectra, and the offshore wind directions. Mitsuyasu et al. (1975) have proposed the relations

$$
\frac{s}{s_{m}}=\left(\frac{f}{f_{m}}\right)^{-2.5} \text { for } f \geqslant f_{m},
$$

where

$$
\frac{s}{s_{m}}=\left(\frac{f}{f_{m}}\right)^{5} \quad \text { for } f \leqslant f_{m},
$$

$$
s_{m}=11.5\left(\frac{U \omega_{m}}{g}\right)^{-2.5}, \quad \omega_{m}=2 \pi f_{m},
$$

so that

$$
s=11.5\left(\frac{U}{c}\right)^{-2.5} \text { for } f \geqslant f_{m} .
$$

Based on the argument that primarily nonlinear processes determine the wave spectrum, Hasselmann et al. (1976) have suggested that $s$ should depend mainly on $f / f_{m}$, rather than on $U / c$ for $f \geqslant f_{m}$. As a referee has pointed out, the spread parameter $s$ might be expected to depend both on $U / c_{m}$ and $f / f_{m}$ even if the directional distribution is mainly governed by nonlinear transfer, since the peak enhancement parameter $\gamma$ and Phillips' "constant" $\alpha$ for the 1-D JONSWAP spectrum (Hasselmann et al., 1973) depend on $U / c_{m}$. Nevertheless, the general tendency should be as follows: if the spectral shape was entirely governed by input from the wind, the relation $s=s\left(U / c_{m} ; f / f_{m}\right)$ should degenerate into $s=s(U / c)$; if, on the other hand, the spectral shape was governed by nonlinear interactions, we should expect $s$ to depend mainly on $f / f_{m}$, with a slight dependence only on $U / c_{m}$. In Figs. 2 and 3 we show our values of $s_{1}$ plotted against either $f / f_{m}$ or $U / c$, and in keeping with (4.4) we have used the deepwater value for $c$ throughout. The large scatter does not allow a decision for the one or the other parameterization based solely on the correlation coefficient. But a grouping into the classes I, II, III and IV shown in Table 2 clearly shows the superiority of the $f_{m}$ scaling. 


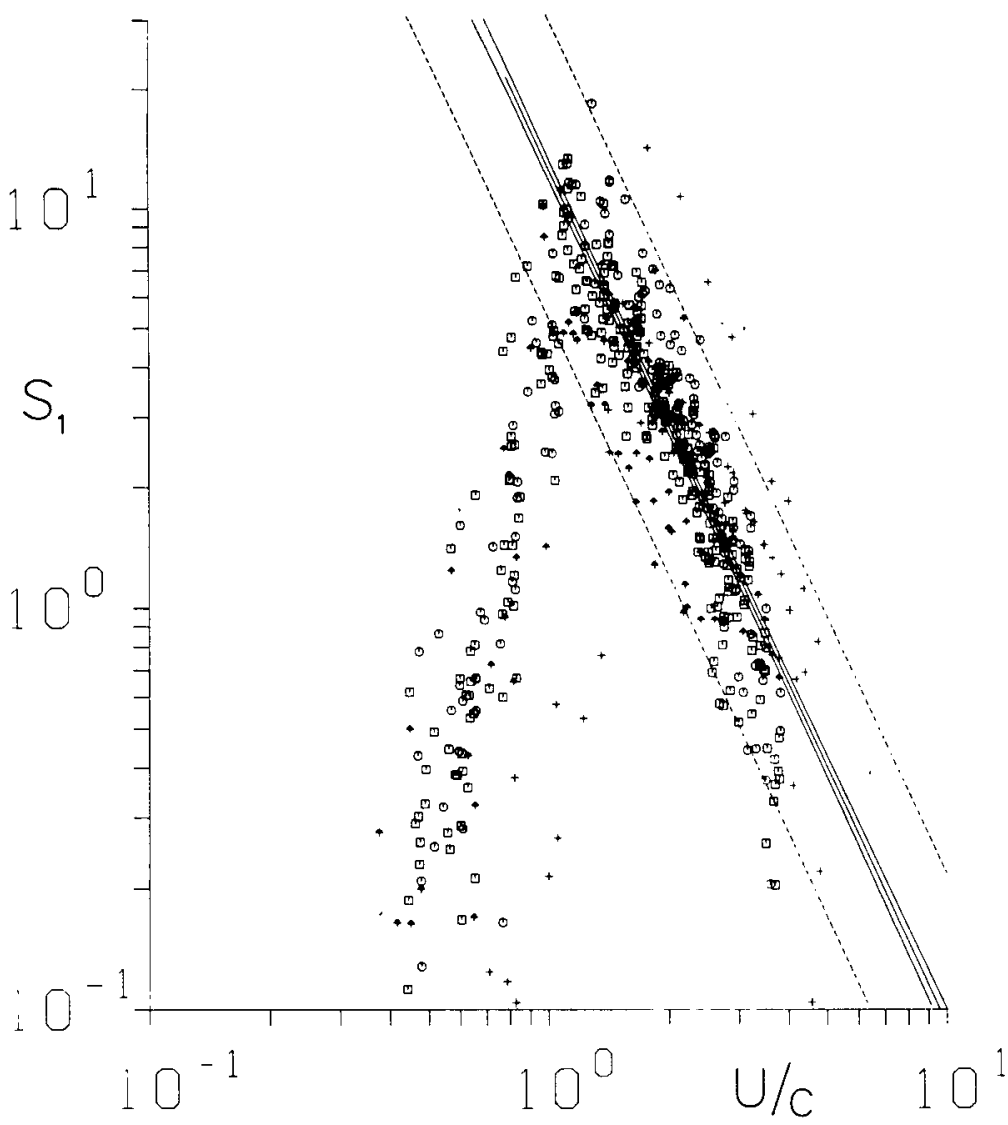

Fig. 2. Spread parameter $s_{1}$ vs $U / C$ for data set A. Symbols classify data by station and group: (I) - (III) of Table $2 ; \square(8, \mathrm{I}), \bigcirc(8, \mathrm{II}),+(8, \mathrm{III}), \uparrow(10, \mathrm{I})$. Also, shown are the regression line for group IV, the $95 \%$ limits for the mean values and the standard deviation around the regression.

We see a significant (always at the $5 \%$ confidence level) increase of $a$ with $U / c_{m}$, whereas $A$ remains constant. As expected, $b=B$ within statistical error, but both parameters show a significant decrease with $U / c_{m}$, so that with increasing $U / c_{m}$ and constant $f / f_{m}>1$ the angular distributions become more isotropic. It is easily estimated (see also Section 5) that the scatter around the regression line is much larger than could be explained by sampling error at $\nu=150$ degrees of freedom. Thus hidden parameters or "geophysical variability" determine this scatter.

In subclass I our range of $U / c_{m}\left(1.0 \leqslant U / c_{m} \leqslant 1.2\right)$ overlaps with that considered by Mitsuyasu et al. (1975). Our values for $a$ and $b$ are close to and presumably in statistical agreement with their values (for which error margins were not given).

From the results of Table 2 we obtain as our final best estimate

where

$$
s_{1}=s_{m}\left(f / f_{m}\right)^{\mu},
$$

$s_{m}=9.77 \pm 0.43$,

$$
\begin{aligned}
\mu=-(2.33 & \pm 0.06) \\
& -(1.45 \pm 0.45)\left(U / c_{m}-1.17\right) .
\end{aligned}
$$

This estimate has been derived from the data for $U \geqslant c_{m}$ and $f \geqslant f_{m}$; the errors are standard deviations. From the data $U \geqslant c_{m}$ and $f<f_{m}$, we obtain a best estimate

with

$$
s_{1}=\tilde{s}_{m}\left(f / f_{m}\right)^{\tilde{\mu}},
$$

$$
\begin{aligned}
\tilde{s}_{m} & =6.97 \pm 0.83, \\
\tilde{\mu} & =4.06 \pm 0.22 .
\end{aligned}
$$

The values for $s_{m}$ and $\bar{s}_{m}$ are significantly different from each other and the simplest way to obtain a consistent parameterization is to shift the breakpoint $f=f_{m}$ to $f=1.05 f_{m}$, so that (4.6) is valid for $f \geqslant 1.05 f_{m}$ and (4.7) for $f \leqslant 1.05 f_{m}$. It is evident from Fig. 3 that the regression line (4.6) systematically overpredicts $s_{1}$ for $f / f_{m} \geqslant 3.0$, but we have made no attempt to determine a higher order regression.

We stress that our data base is not ideal and more measurements in ideal generation conditions and at 


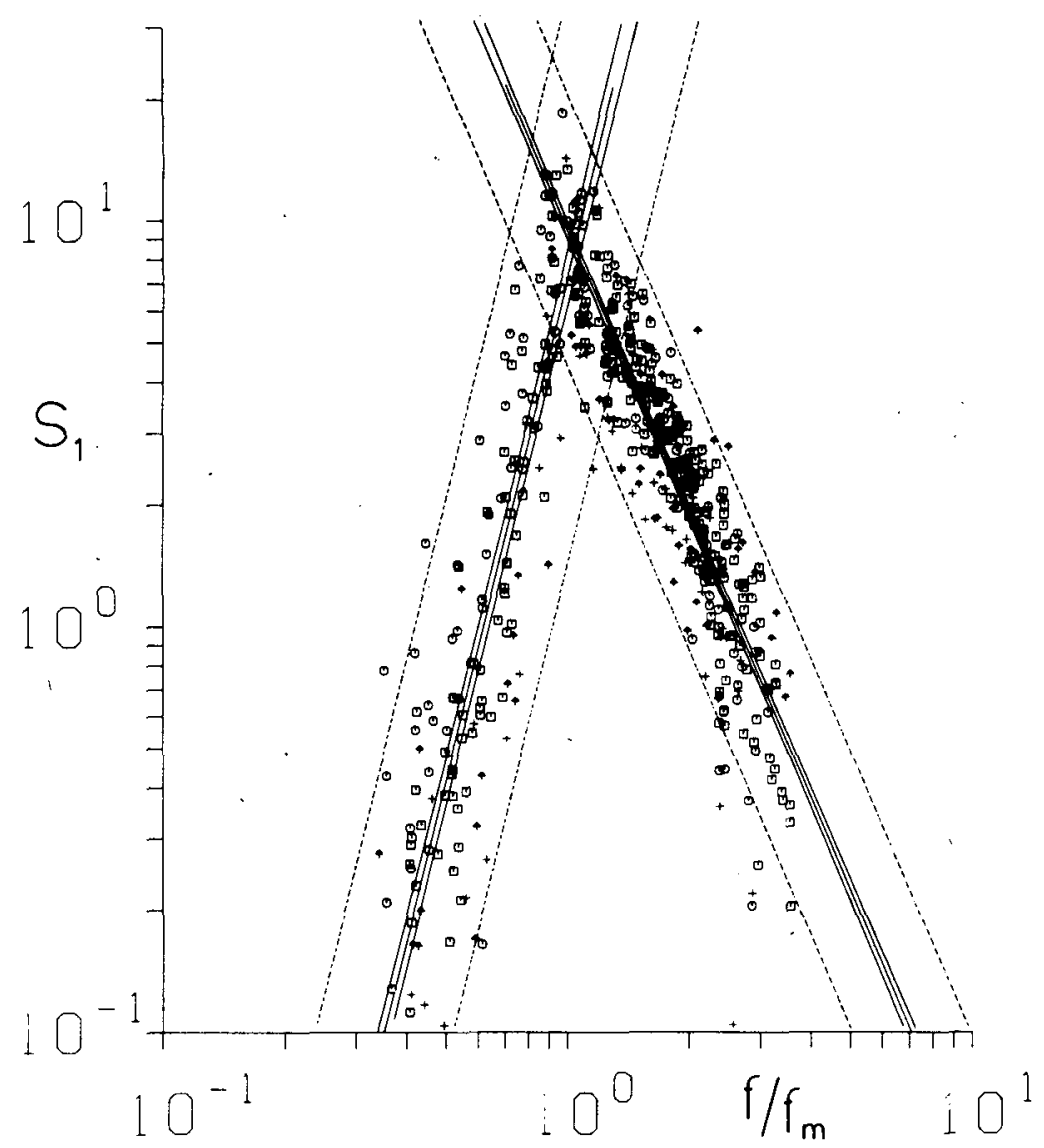

Fig. 3. As in Fig. 2 except $s_{1}$ vs $f / f_{m}$ for data set A.

small nondimensional fetch would be helpful to check our conclusions.

Whether our parameterization (4.6) and (4.7) remains valid in the nearly fully developed growth range $0.8 \leqslant U / c_{m}<1$ remains to be seen. The high $s$ values reported by Mitsuyasu et al. (1975) provide evidence to the contrary, but it should be kept in mind that in this range of $U / c_{m}$ it is often difficult to distinguish between swell and sea; for this reason we have not included data from this range in our analysis. For large values of $U / c_{m}$ some results supporting our conclusion that (4.5) needs improvement have been obtained from experiments in sufficiently wide tanks at $U / c_{m} \approx O(10)$ when the directional spread was found to be narrower than given by (4.5) (preliminary information kindly provided by a referee and by $\mathrm{M}$. Donelan).

If $S_{1}(f, \theta)$ of (4.1)-(4.3) is a good approximation of $S(f, \theta)$, then $s_{2}=s\left(r_{2}\right)$ should lie close to $s_{1}$ where (Cartwright, 1963)

$$
r_{2}=\frac{s_{2}\left|s_{2}-1\right|}{\left(s_{2}+1\right)\left(s_{2}+2\right)} .
$$

For $0 \leqslant s_{1} \leqslant 1$ the root $s_{2}$ of (4.8) closest to $s_{1}$ is understood. In Fig. 4 we show the comparison
TABLE 2. Regression analysis, Data set A.

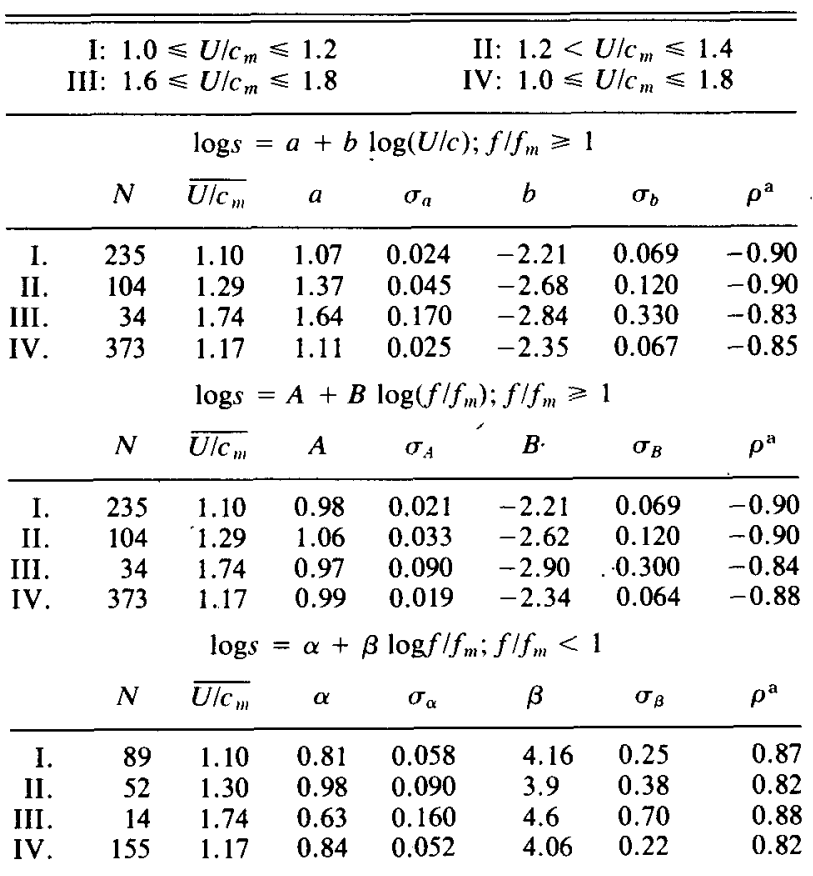

a Correlation coefficient. 


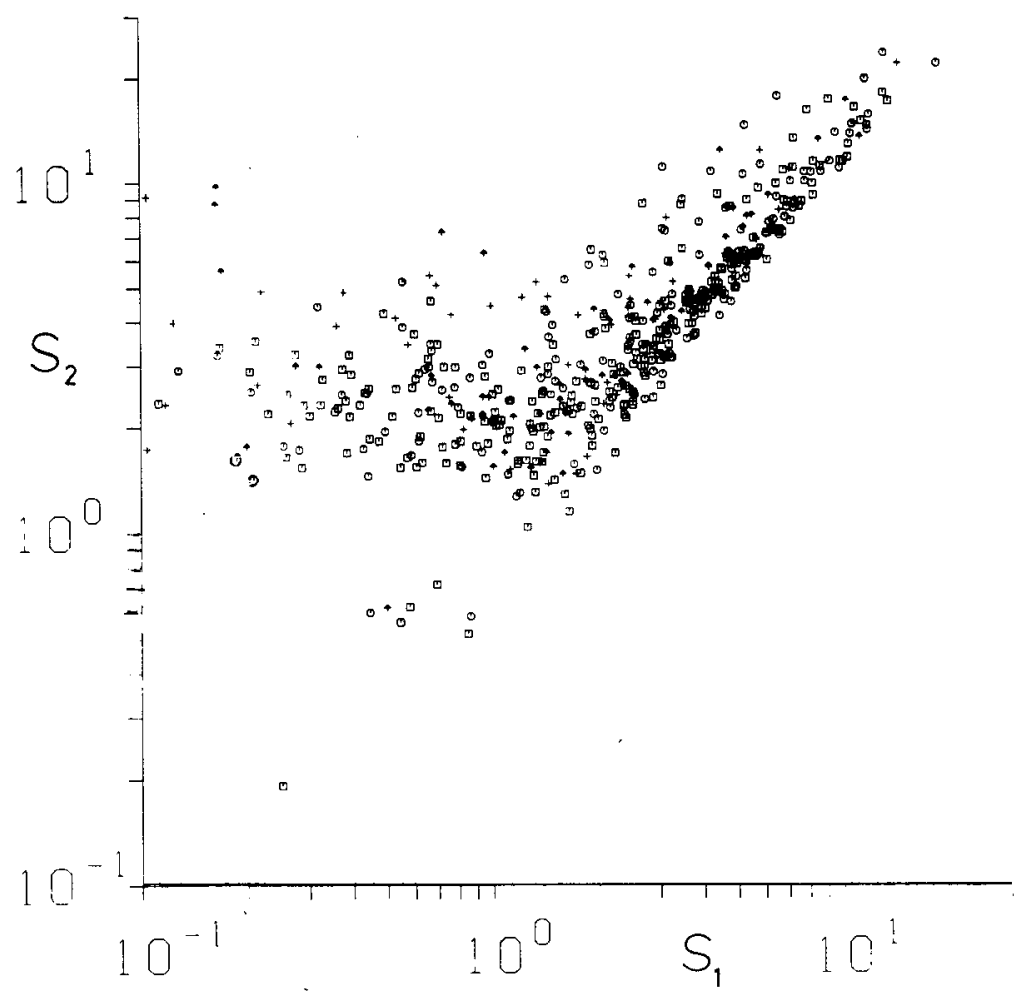

FIG. 4. As in Fig. 2 except $s_{1}$ vs $s_{2}$ for data set A.

between $s_{1}$ and $s_{2}$, and while there is clearly a high correlation for $s_{1}>3$, considerable scatter at lower values of $s_{1}$ is also evident.

At near isotropic distributions $s_{1}<1$ the sampling error will cause a bias of the estimator $\hat{r}_{2}$ at $\nu$ degrees of freedom in order of $(1 / \nu)^{1 / 2} \approx 0.08$ at $\nu=150$. In $0 \leqslant s \leqslant 1$ the maximum value of $r_{2}(s)$ is $r_{2}=0.072$ [at $s=(\sqrt{ } 3-1) / 3$ ] and thus in most cases the bias will enforce a value $s_{2}>1$. Thus we have a simple explanation for most of the points with $s_{1}<1$ in Fig. 4, but not for those with $s_{2}>3.0$, $s_{1}<1$.

\section{Improved fits and testing of hypotheses}

\section{a. Improved fits}

In the comparatively simple conditions of data set A the fit (4.1) would seem to provide a reasonable description of the angular distribution. In more complicated cases, when there was a swell running into a windsea or especially when the wind was turning the simple $1 \mathrm{M}$ fit was clearly not appropriate and the need for a fit which included all the information arose.

Two methods of fitting were therefore investigated: the maximum likelihood (ML) fit of Long and Hasselmann (1979), denoted with $S_{2}(\theta)$, and the $2 \mathrm{M}$ fit, which has the form

$$
S_{3}(\theta)=\lambda M\left(\theta, p_{1}, \beta_{1}\right)+(1-\lambda) M\left(\theta, p_{2}, \beta_{2}\right),
$$

with $1 / 2 \leqslant \lambda \leqslant 1$ and for uniqueness we impose the side condition $\left(p_{1}-p_{2}\right)^{2}=$ min; details are given in Appendix B. Uniqueness of the ML fit $S_{2}(\theta)$ is ensured by requiring minimum distance to a most preferred function $\hat{S}_{2}(\theta)$, i.e.,

$$
\int\left[S_{2}(\theta)-\hat{S}_{2}(\theta)\right]^{2} d \theta=\min
$$

and for all of our cases we have chosen $\hat{S}_{2}=S_{1}$ from $(4.1)$.

In the versions we used both the ML fit and the $2 \mathrm{M}$ fit reproduce the Fourier coefficients exactly and make no allowance for statistical error. We therefore refer to them as exact fits.

We have looked at the following questions:

1) How well do the fitting procedures reproduce known artificial angular distributions when they have only the four lowest Fourier coefficients as input information and how do they behave numerically?

2) How do the algorithms respond numerically to measured Fourier coefficients?

3) To which degree do the Fourier coefficients determine the angular distribution and can we define a typical distance between exact fits?

4) Does allowance for statistical error drastically increase a typical distance between two allowed fits 


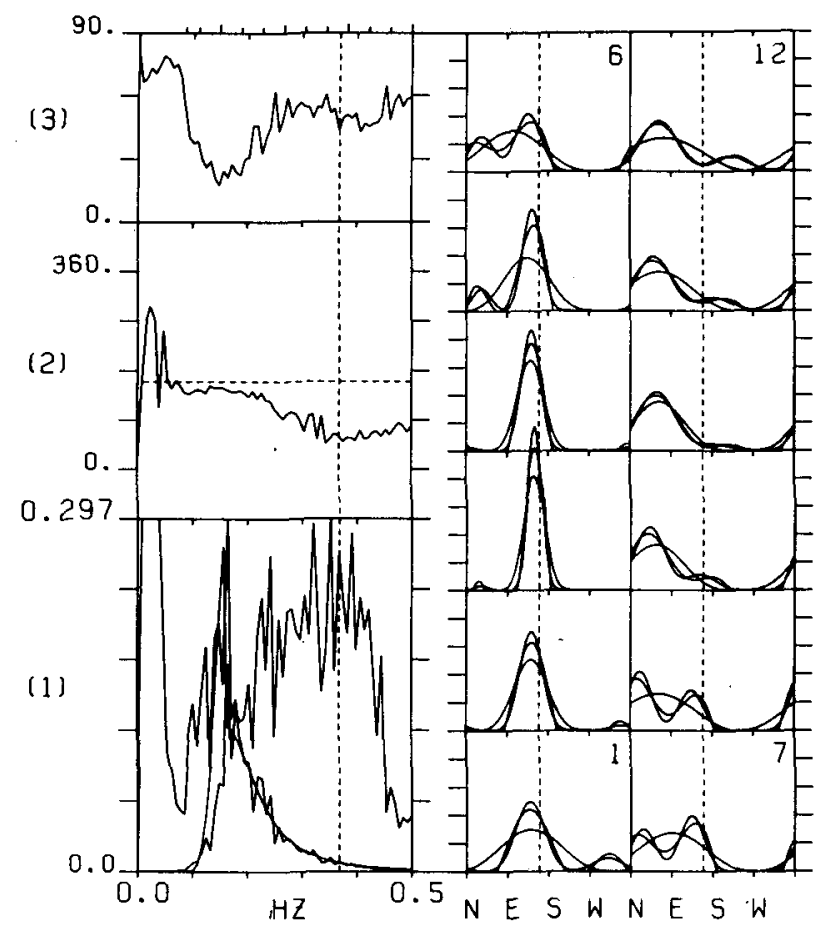

Fig. 5. Spectra for case B3 (day 256, time 2000, station 8). LEFT: Boxes (1), (2), (3) show $E(f), \delta_{1}(f)$ and the mean-square spread angle $\theta_{2}(f)$, respectively. Also indicated by dashed lines are the mean wind direction $\delta_{w}$ and the frequency $f_{u}$ at which $U=c$, $U=g / 2 \pi f_{u}$. In box (1) $E(f)$ shows twice-integrated lowfrequency noise to about $0.08 \mathrm{~Hz}$ and then rises to a maximum of $0.297 \mathrm{~m}^{2} \mathrm{~Hz}^{-1}$ at $0.17 \mathrm{~Hz}$. Also shown is the JONSWAP fit $\tilde{E}$ to $E(f)$ with a slightly lower peak frequency and vanishing below $0.1 \mathrm{~Hz}$. The third curve, rising from $0.1 \mathrm{~Hz}$ and causing some confusion at $0.17 \mathrm{~Hz}$, where it crosses over onto its plateau for $0.2 \leqslant f \leqslant 0.4 \mathrm{~Hz}$, is $A(f)=f^{5} E(f)$. The scale for $A(f)$ runs from 0 to $0.0002 \mathrm{~m}^{2} \mathrm{~Hz}^{4}$. RIGHT: Directional distribution functions $S_{1}(\theta), S_{2}(\theta)$ and $S_{3}(\theta)$ for bands $1, \ldots, 12$. [The frequencies $f_{n}$ are indicated by the frequency tick marks on top of the upper left box (3), $f_{1}=11 / 128 \mathrm{~Hz}, f_{n}=(5 n+4) /$ $128 \mathrm{~Hz}$ for $n>1$.] The $1 \mathrm{M}$ fit $S_{1}(\theta)$ is in all cases the broadest distribution, while the $2 \mathrm{M}$ fit $S_{3}(\theta)$ is always that with the highest maximum value. At all frequencies the scale is from 0 to 1.25 . The $1 \mathrm{M}$ fit is accepted in bands 6,7 and 10 . The maximum values of $\Delta_{1,2}$ and $\Delta_{2,3}$ are $\Delta_{1,2}=0.99$ in band 3 and $\Delta_{2,3}=0.51$ in band 5 .

as compared to the distance between two exact fits? This point will be examined in Section $5 \mathrm{~b}$.

The other points are answered as follows:

(i) In many trials on artificial data we found both methods to be fast and stable, but the ML fit is more stable. The results of the ML fit do not sensitively depend on the choice of $\hat{S}_{2}(\theta)$. Both fits reproduced input distributions with at most two peaks (e.g., two boxes) surprisingly well. When there were more than two peaks both methods were unable to reproduce this structure and returned at most two peaks for $\hat{S}_{2}=S_{1}$ or $\hat{S}_{2}=1 / 2 \pi$.

(ii) When applied to our 194 runs, yielding 2328 fits, the ML fit failed in only 16 cases, all of which occurred in two runs, while the $2 \mathrm{M}$ fit failed in 132 cases. The concentration of ML errors in two runs strongly suggests that these data were erroneous and we have found the ML fit to provide an efficient means of checking the validity of data.

(iii) The results of the two fits were often very close together, even when they deviated markedly from the $1 \mathrm{M}$ fit. As a measure of distance we use a normalized form of (5.2):

$$
\Delta_{i, j}^{2}=4 \pi \int_{0}^{\infty}\left(S_{i}-S_{j}\right)^{2} d \theta
$$

so that $\Delta=1$ is the distance between isotropy and $(1+\cos n \theta) / 2 \pi$.

Good agreement between fits is usually obtained for $\Delta<0.2$ (e.g., see Figs. 5 and 6). In more than 95\% of all cases the distance between exact fits fell into the range $\Delta_{2,3}<0.4$.

The frequency distributions of the distances $\Delta_{1,3}$ and $\Delta_{1,2}$ to the $1 \mathrm{M}$ fit were nearly identical [in agreement with (5.2) $\Delta_{1,2}<\Delta_{1,3}$ always held] and only in $53 \%$ of the cases was $\Delta_{1,2}<0.4$. It is often stated that pitch-and-roll buoys have only a coarse angular resolution. The results of (i)-(iii) allow us to qualify this statement:

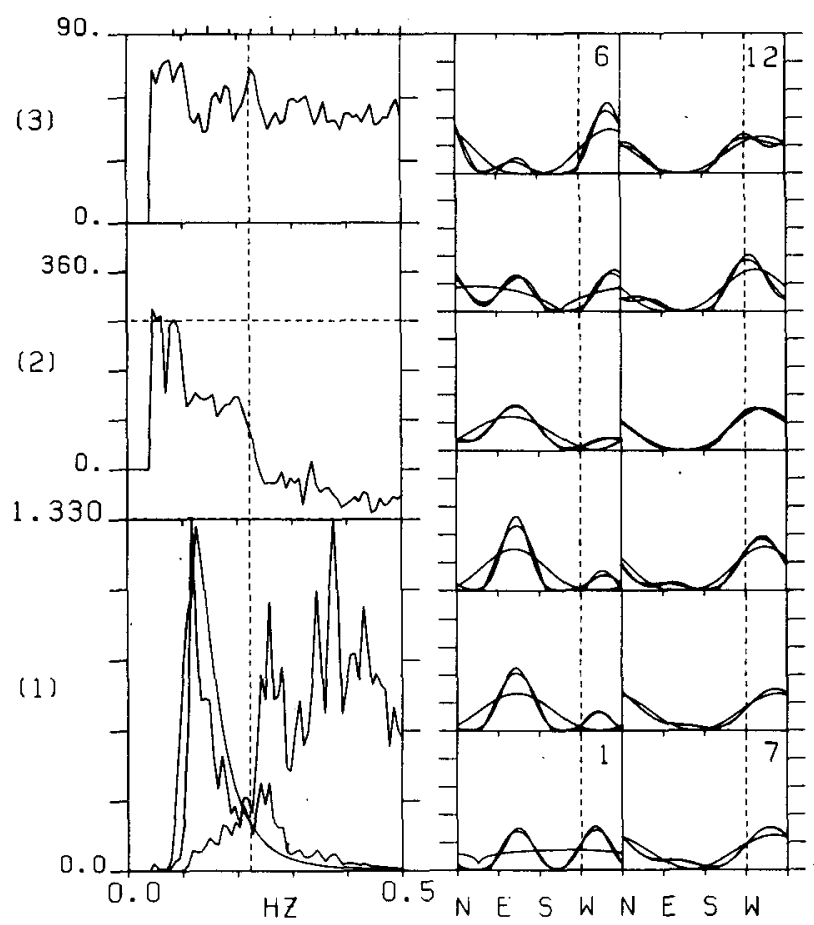

FIG. 6. Spectra for case B4 (day 264, time 0815, station 10). LEFT: As in Fig. 6 except that $E, \tilde{E}, A$ are now easily distinguishable. The scale for $E$ and $E$ is from 0 to $1.33 \mathrm{~m}^{2} \mathrm{~Hz}^{-1}$ and for $A$ from $0-0.00037 \mathrm{~m}^{2} \mathrm{~Hz}^{-4}$. RIGHT: Same scale and the same features identify $S_{1}, S_{2}$ and $S_{3}$ as in Fig. 5. (Due to finite plot steps the $1 \mathrm{M}$ fit in band 1 does not have $S_{1}=0$ at NE as it should.) The $1 \mathrm{M}$ fit is accepted in bands 8,10 and 12 . The largest distances $\Delta$ are $\Delta_{1,2}=1.12$ and $\Delta_{2,3}=0.34$, both found in band 3 . 
TABle 3. Percentage contributions for model 1 $(N=2304$; entire data set).

\begin{tabular}{lrcc}
\hline \hline & $0 \leqslant \Delta$ & $\Delta<0.4$ & $\Delta \geqslant 0.4$ \\
\hline $0 \leqslant \chi^{2}$ & 100 & 53 & 47 \\
$\chi^{2}<5.99$ & 57 & 50 & 7 \\
$\chi^{2} \geqslant 5.99$ & 43 & 3 & 40 \\
\hline
\end{tabular}

If all available information is taken into account then pitch-and-roll buoys have a high resolving power for angular distributions consisting of one or two narrow peaks and will not tend to smear these out. If, for instance, the true angular distribution is the $\delta$ function, $S(\theta)=\delta(\theta)$, or if $S(\theta)=0.5[\delta(\theta)$ $+\delta(\theta-\pi)]$, then the only fit to pitch-and-roll information is the true distribution; and if the true angular distribution is close to these examples then any fit must remain close to these.

As the true distribution becomes more isotropic the positivity constraint $S \geqslant 0$ becomes less stringent, so that for broad angular distributions the angular resolution of a pitch and roll buoy is indeed coarse.

The sampling error has the same tendency: It vanishes for the first $\delta$ function example given above and is not important for distributions close to either example; for nearly isotropic distributions, however, the bias for $r_{1}$ and $r_{2}$ may produce spurious peaks (see, for example, the lower frequency bands in Figs. 5 and 6).

\section{b. Testing of hypotheses}

We have not yet considered whether the $1 \mathrm{M}$ fit, despite its often large distances to exact fits, is nevertheless statistically consistent with the data. Nor have we considered, whether the parameterization of Section 4 is in statistical agreement with the data, although the abovementioned large scatter around the regression makes this unlikely. The con- cept of minimizing $\chi^{2}$ (Müller et al., 1978; Long and Hasselmann, 1979; Long, 1980) allows us to find a best model (with a given number of parameters) and the minimum $\chi^{2}$ then allows us to decide whether the best model can be accepted. We have not attempted to find a best model, but have simply tested three obvious models for consistency with the data. A model is rejected at the $95 \%$ confidence level if $\chi^{2}>\chi_{\nu_{1} ; 0.05}^{2}$ where $\nu_{1}$ is the number of predicted variables.

Model 1 is our $1 \mathrm{M}$ fit and has $\nu_{1}=2$ since only $r_{2}$ and $\delta_{2}$ are predicted.

Model 2 is given by $S(\theta)=M\left(\theta, s, \delta_{w}\right)$, where $s$ is taken from the $U / c$ dependent parameterization group IV in Table 2 for $f \geqslant f_{m}$; for $f \leqslant f_{m}$ we have chosen $s / s_{m}=\left(f / f_{m}\right)^{4}$ with $s_{m}$ depending on $U / c_{m}$.

Model 3 is given by $S(\theta)=M\left(\theta, s, \delta_{w}\right)$ where we have now used $s$ as given by the $f / f_{m}$ dependent parameterization group IV in Table 2 , but with the breakpoint at $f / f_{m}=1.05$.

For models 2 and $3 \nu_{1}=4$, since all parameters $r_{1}, \delta_{1}, r_{2}, \delta_{2}$ are predicted variables.

We have tested the last two models only against the runs of data set A. Details are given in Appendix C.

Model 1 was rejected in $28 \%$, model 2 in $83 \%$ and model 3 in $82 \%$ of these cases. We did not test our best parameterization [(4.6) and (4.7)], but the results would undoubtedly have been similar. Thus, the poor prediction of the first harmonic $\left(r_{1}, \delta_{1}\right)$ is mainly responsible for the inconsistency of models 2 and 3 , since model 1 is consistent in the majority of cases.

To see how well the distance $\Delta=\Delta_{1,2}$ corresponds to the consistency of fits, we have compiled Table 3 , which shows that $57 \%$ of all $1 \mathrm{M}$ fits were consistent with the data, and nearly all of these fell into the range $\Delta<0.4 ; \Delta_{2,3}=0.4$ presents an upper bound for the typical distance between exact fits [see (iii) of Section 5a]. Conversely if $\Delta \geqslant 0.4$, nearly all $1 \mathrm{M}$ fits were inconsistent with the data.

Thus, for pitch-and-roll measurements there is

TABle 4. Data Sets B and B' $\left(B^{\prime}=B-B 5\right)$.

\begin{tabular}{|c|c|c|c|c|c|c|c|c|}
\hline Case & $N_{\text {run }}$ & $\begin{array}{l}\text { Sta- } \\
\text { tion }\end{array}$ & Day number/time & $\begin{array}{c}U^{\mathrm{a}} \\
\left(\mathrm{m} \mathrm{s}^{-1}\right)\end{array}$ & $\delta_{w}^{a}$ & $\dot{\delta}_{u t}^{\mathrm{b}}$ & Swell ${ }^{\mathrm{c}}$ & Response $^{d}$ \\
\hline B1 & 13 & 8 & $250 \quad 1500-251 \quad 900$ & $8-4$ & $120-060$ & $-60 / 5$ & + & h \\
\hline \multirow[t]{2}{*}{$\mathrm{B} 2$} & 44 & 8 & $2532000-2560400$ & $12-8$ & $160-130-160$ & $\pm 30 / 12$ & + & $\mathrm{mh}$ \\
\hline & & & & $8-4$ & $160-320$ & $160 / 10$ & + & $\mathrm{h}$ \\
\hline B3 & 11 & 8 & $2561800-2570600$ & $4-6$ & $60-160-120$ & $90 / 1$ & + & $\mathrm{mh}$ \\
\hline B4 & 6 & 10 & $2632000-264 \quad 1200$ & $4-12$ & $60-(-100)$ & $-150 / 12$ & + & $\mathrm{mh}$ \\
\hline B5 & 13 & 8 & $2642200-2651200$ & $8-2-10$ & $310-150$ & $-160 / 6$ & + & non-locale \\
\hline B6 & 14 & 8 & $2671800-2681100$ & $8-12-8$ & $330-240$ & $-90 / 5$ & - & $\mathrm{mh}$ \\
\hline B7 & 24 & 8 & $268 \quad 1800-2692000$ & $10-4$ & $210-320$ & $110 / 9$ & + & $\mathrm{mh}$ \\
\hline
\end{tabular}

a Range gives crude temporal information.

b Typical values in degrees $\mathbf{h}^{-1}$.

c Plus values indicate spectrum dominated by swell.

d Response of $\delta$, in high (h), or medium (m) bands.

* Not included in the data set B' selected for further analysis. 

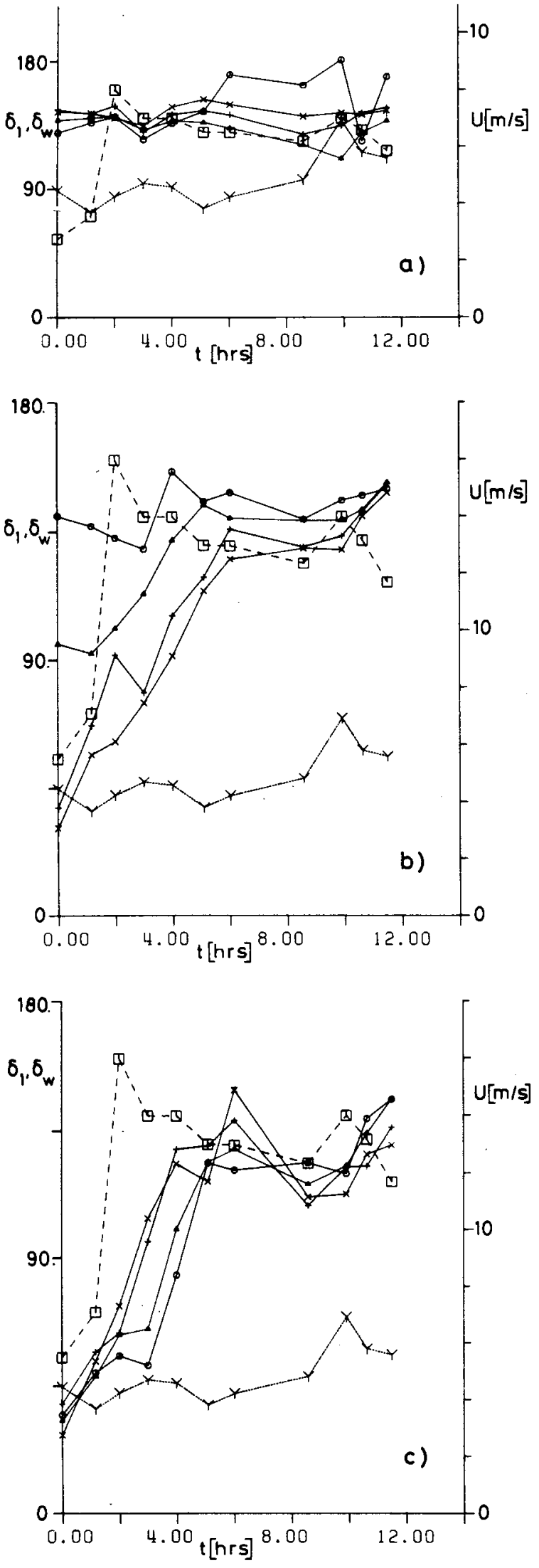

little point in increasing the number of degrees of freedom beyond $\nu=150$, since then the distance between exact fits and not the sampling error becomes the limiting element in data interpretation.

\section{The response to veering winds \\ a. Presentation of the data}

The directional distribution in the case of veering winds can be rather complex, since a number of competing mechanisms are acting:

(i) the local wind will have a tendency to align the waves in the wind direction.

(ii) Turning winds are usually associated with a nonhomogeneous wind field, so that the wind, say, some $50 \mathrm{~km}$ away, might be generating waves which are radiated into our area in a direction deviating from that of the local wind.

(iii) Interactions with swell will have some, although normally rather weak, tendency to align the entire wave field into the swell direction.

(iv) In our site shallow water refraction effects may play a role.

We have considered only the influence of mechanism (i), unless there was clear evidence that a case was dominated by other processes-such cases were not included in data set $B^{\prime}$ selected for the further analysis of Section $6 \mathrm{~b}$.

We discuss here only the behavior of $\delta_{1}$, since of all variables $\left(r_{1}, r_{2}, \delta_{1}, \delta_{2}\right.$ or $\left.s_{1}, s_{2}, \delta_{1}, \delta_{2}\right) \delta_{1}$ was the only one to show a clearly detectable response to the wind. The response of $r_{1}$, or the mean square spread angle $\theta_{2}$ was sometimes detectable, but was much harder to separate from the noise.

The runs for data set B (Table 4) were selected solely by the criterion that the wind was turning, so that we have purposely avoided a bias toward "strong effects." The decision whether a wind was turning or just fluctuating was made subjectively. Times prior to the turning of the wind were sometimes included and thus there is some overlap with data set $\mathrm{A}$. These runs are marked by a footnote in Table 1. All other runs of data set B had a strong or dominant swell contribution.

For lack of space we can present a detailed analysis only for cases B3, B4 and B7. On the basis of such an analysis the data of case B5 (Günther et al., 1979) were seen to be dominated by mechanism (ii) mentioned above. These data were the only ones not included in the selected set $\mathrm{B}^{\prime}$.

Fig. 7. Case B3 (station 8 ); $t=0$ corresponds to day 256 at 1800. Shown are the wave directions $\delta_{1}$, the wind direction $\delta_{w}(\square)$ and the wind speed (Y) as function of time for the lowfrequency (a), medium-frequency (b) and high-frequency bands (c). Bands $1-4,5-8$ and $9-12$, respectively, have symbols $O$, $\triangle,+, x$. 


\section{1) Case B3 (steplike change)}

In Fig. 7, we show the development in time of $\delta_{w}, U$ and $\delta_{1}$ for the low-, medium- and high-frequency bands. During day 256 the wind $U=4-6$ $\mathrm{m} \mathrm{s}^{-1}$ had shifted from $\delta_{w}=270^{\circ}$ at 0400 (all times GMT) through north to $\delta_{w}=60^{\circ}$ at 1800 (see Fig. 1). At the last previous measurement $(256,0400)$ we observed $\delta_{1}=150^{\circ}$ for the bands $1, \ldots, 8$, while for the high-frequency bands $\delta_{1}$ ran from $\delta_{1}=190^{\circ}$ for band 9 to $\delta_{1}=270^{\circ}$ at band 12 .

Comparison with the values at 1800 shows that the medium- and high-frequency bands had responded to the shifting winds and that band 5 behaves as swell, whereas the value of $\delta_{1}$ in band 6 can be explained by a strong swell component. Between 1900 and 2000 the wind shifted rapidly from $70^{\circ}$ to $160^{\circ}$ and then gradually fell back to $120^{\circ}$. We see that both the medium- and high-frequency bands responded and that the slope $\dot{\delta}_{1}$ is greater for the high-frequency than for the medium-frequency bands.

The wave spectrum was at all times dominated by swell; Fig. 5 is typical for the one-dimensional spectrum, which also shows that the swell tail extends to band 8 and that the medium-frequency bands still contain a strong northerly component due to the history before 1800 , while the high-frequency bands reflect the prevailing wind direction between 1800 and 1900 .

The secondary dip in $\theta_{2}$ is typical for turning wind situations, but its position is not normally well defined and the dip is usually undetectable when we plot $\theta_{2}(f, t)$ at a fixed frequency. There is no detectable increase of the wave spectrum in the generating range $U \geqslant c$ and this feature was found in all cases except the next one B4.

\section{2) Case B4 (constant angular velocity)}

Fig. 8 shows a beautiful example of a wind turning at nearly constant angular velocity-over a period of $12 \mathrm{~h}$ the wind turned from $\delta_{w}=60^{\circ}$ through north to $\delta_{w}=-90^{\circ}$. The wind speed increased from 4 to $11 \mathrm{~m} \mathrm{~s}^{-1}$, which is the reason for the increase in the one-dimensional wave spectrum in the frequency range $U \geqslant c$. Nevertheless, the spectrum was always dominated by swell (see Fig. 6).

The response of $\delta_{1}$ is absent in the swell-dominated low-frequency bands but is clearly evident in the medium- and high-frequency bands and now we observe fairly good agreement between the slopes $\dot{\delta}_{1}$ and $\dot{\delta}_{w}$.

We notice in Fig. 6 that the mean spread angle $\theta_{2}$ is large compared to the steady wind cases. At the end of the period when the wind steadies $\theta_{2}$ decreases (not shown). Although this has also occasionally been observed in other cases, we have not been able to establish a definite correlation between $\theta_{2}$ and $\dot{\delta}_{w}$ for the entire data set B.
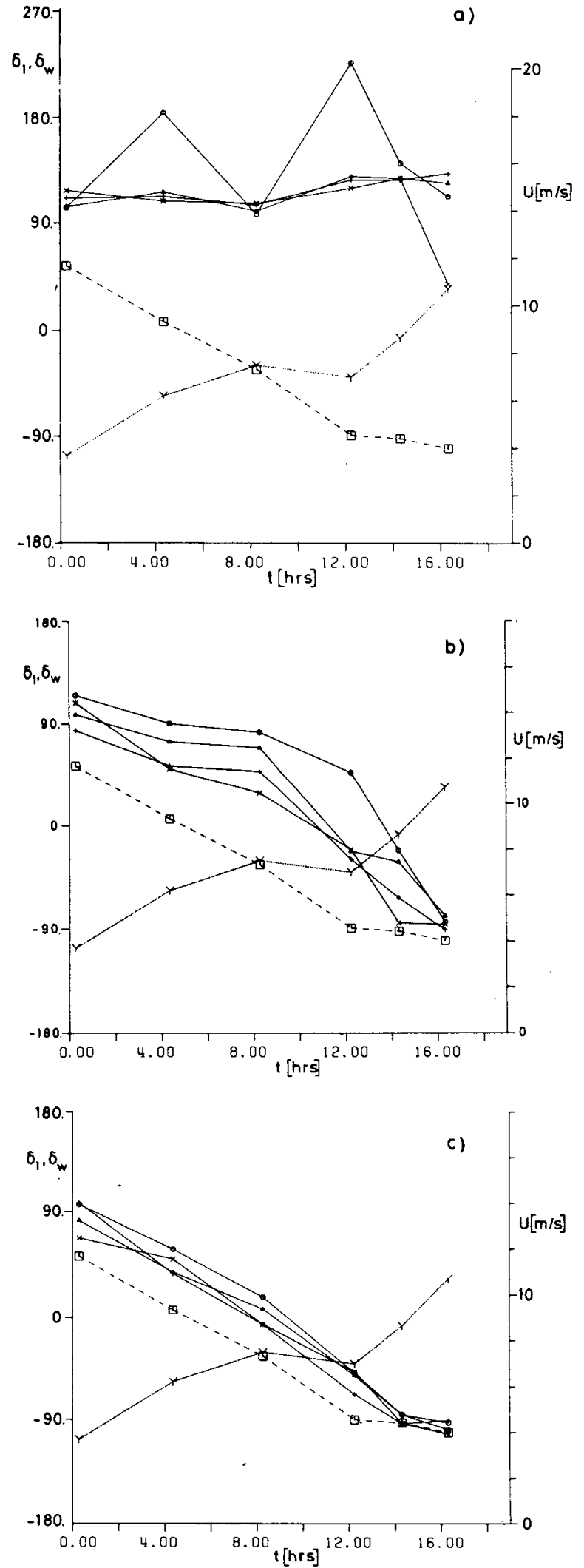

FIG. 8. Case B4 (station 10); $t=0$ corresponds to day 263 at 2000. Otherwise as in Fig. 7. 

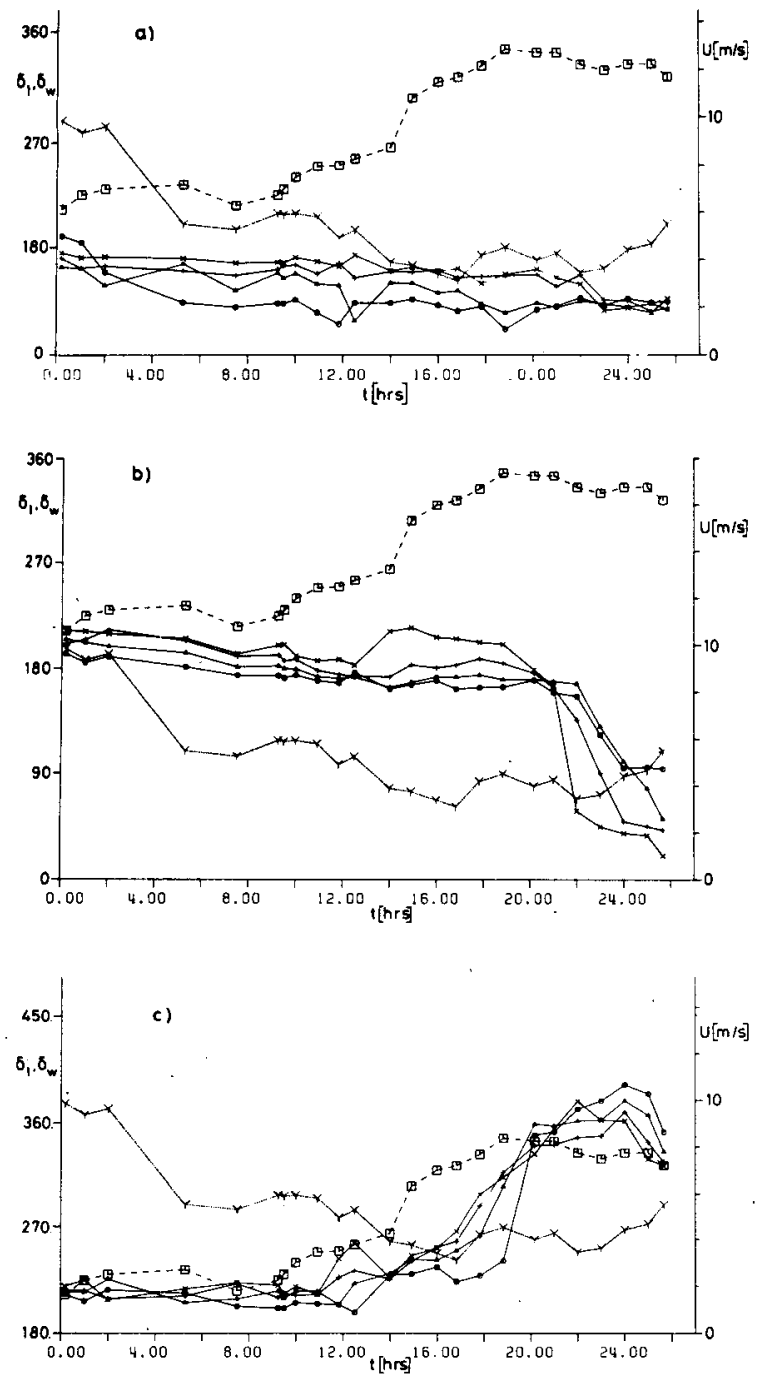

FIG. 9. Case B7 (station 8); $t=0$ corresponds to day 268 at 1800. Otherwise as in Fig. 7.

\section{3) CAse B7 (mixed situation)}

This case is more complicated than the previous two. Between 269 at 0200 and 269 at 1300 the wind gradually turned from $\delta_{w}=210^{\circ}$ to $\delta_{w}=310^{\circ}$ at an approximately constant angular velocity. The wind speed was only $3.5-6 \mathrm{~m} \mathrm{~s}^{-1}$ (see Fig. 9). The lowfrequency swell bands again did not respond. The medium-energy bands did not respond until 269 at 1300 when they gradually began to turn into the prevailing wind direction, but interestingly their direction of turn is opposite to that of the wind. Since the wind and wave directions were $\sim 180^{\circ}$ apart before turning this is not too surprising and a swell contribution could easily cause such a behavior. Of these medium-frequency bands, only the highest fell into the range $U \geqslant c$, so that we were possibly observing waves radiated into our area, although the fact that the higher frequencies in the mediumfrequency bands turn faster than the lower ones does not support this interpretation.

We see that the high-frequency bands behave as expected, the highest frequencies being the first to turn. During the time in which the medium-frequency bands are turning the high-frequency bands show a perplexing "overshoot" over the local wind direction, running into a direction intermediate between the wind direction and the direction of the mediumfrequency bands. Perhaps interactions between the medium- and the high-frequency bands are important in this situation.

\section{b. Further analysis of data set $B^{\prime}$}

The type of situation we shall try to model is the following: We consider the response of a homogeneous wind sea to a wind field which is homogeneous, but changes direction $\delta_{w}=\delta_{w}(t)$ while the speed remains constant. This is an idealization which is never met in the field. However, neither does our data base allow a reasonable estimate of horizontal scales of the wind and wave fields, nor does a simple theoretical framework exist which could easily incorporate horizontal inhomogeneity. Inhomogeneity can be included in numerical models, but this is beyond the scope of this paper. Even in the idealized case it is not simple to decide how the wavefield is expected to behave. We have finally opted for an extremely simple model, aware of the fact that it cannot render a correct description.

We have considered testing models which can incorporate more of the physics, but eventually decided the effort was not worthwhile, since it is doubtful whether our noisy data would allow the determination of a more complex process.

Our first simple model was based on the following assumptions:

- Since, except for case B4 in which there was also a marked increase in wind speed, there is no obvious increase of the one-dimensional spectra due to changes in direction, we neglect all effects which may change $E(f)$.

- Concentrating entirely on $S(f, \theta)$ we assume that, even in situations with a turning wind, the directional distribution $S(f, \theta)$ can be approximated by the $1 \mathrm{M}$ fit $S_{1}(f, \dot{\theta})$, so that we deal only with $r_{1}$ and $\delta_{1}$. Plots of the distributions nearly always showed considerably skewed, often double-peaked distributions, but we have not attempted to describe these effects in our simple model.

Introducing the vectors

$$
\begin{aligned}
& \mathbf{r}_{1}=r_{1}\left(\cos \delta_{1}, \sin \delta_{1}\right), \\
& \mathbf{n}=\left(\cos \delta_{w}, \sin \delta_{w}\right),
\end{aligned}
$$


our first attempt was the model

$$
\dot{\mathbf{r}}_{1}=A \mathbf{n}-B \mathbf{r}_{1},
$$

where the parameters $A$ and $B$ were allowed to depend on $U / c$ and $f / f_{m}$. There is only one unknown for the response to changing winds in (6.2) since the analysis of the data set $A$ prescribes the ratio $A / B$ for $\delta_{w}=$ constant .

According to (6.2), a turning wind causes both a decrease of $r_{1}$ relative to its equilibrium value and an angular adjustment. We have analyzed the data according to (6.2), performing a linear regression between the left-hand and right-hand sides of $(6: 2)$.

Due to large scatter no reasonable estimates could be obtained, except for a poor determination of the known value of $A / B$. Thus we analyzed an even simpler model by locking $r_{1}$ into its equilibrium value, thereby eliminating the scatter of $r_{1}$ from the analysis.

This leads to a formulation

$$
\dot{\delta}_{1}=\omega b \sin \left(\delta_{w}-\delta_{1}\right),
$$

where $\omega=2 \pi f$ and $b=b\left(U / c ; f / f_{m}\right)$. For a wind turning with a constant angular velocity $\Omega=\dot{\delta}_{w}$ and constant wind speed, we obtain, for $\Omega /(\omega b)$ $<1$ and $t \rightarrow \infty$, regardless of the initial values,

$$
\delta_{1}=\Omega(t-\tau)=\Omega t-\delta_{l},
$$

where the lag angle $\delta_{l}$ is given by

$$
\sin \delta_{l}=\Omega /(\omega b) .
$$

Applying this analysis to data set B4 we estimate from Fig. $8 \delta_{l} \approx 80^{\circ}$ for the medium-frequency bands and $\delta_{l} \approx 40^{\circ}$ for the high-frequency bands, and thus with $\Omega=150^{\circ}$ per $12 \mathrm{~h}$ both ranges of bands yield the estimate

$$
b \approx 3 \times 10^{-5} \text {. }
$$

On the other hand, for a steplike change of $\delta_{w}$ from $0^{\circ}$ to $90^{\circ} \mathrm{Eq}$. (6.3) predicts

$$
\tan \left(\frac{\pi}{4}-\frac{\delta_{1}}{2}\right)=e^{-\omega b t}
$$

so that for an angle, of, say $\delta_{1}=70^{\circ}$, we obtain

$$
\omega b T_{70}=1.74 \text {. }
$$

With the above value of $b=3 \times 10^{-5}$ this yields

$$
\begin{array}{ll}
T_{70}=2.5 \mathrm{~h} & \text { for } f=0.5 \mathrm{~Hz} \text { (band 12), } \\
T_{70}=3.6 \mathrm{~h} & \text { for } f=0.34 \mathrm{~Hz} \text { (band } 8 \text { ). }
\end{array}
$$

Comparison with Fig. 7 shows reasonable agreement.

We then performed a one-parameter regression analysis of all data in the set $\mathrm{B}^{\prime}$. For $U / C \leqslant 2.0$ our results are given in Table 5; the errors given for $b$ are standard deviations while those for the correlations are $95 \%$ confidence limits. For $U / c>2.0$ the correlation is consistent with zero. These values of
TABLE 5. Regression analyses according to Eq. (6.3).

\begin{tabular}{lccc}
\hline $\begin{array}{l}\text { Range } \\
\text { of } U / c\end{array}$ & Entries & $\begin{array}{c}b \\
\left(\times 10^{5}\right)\end{array}$ & Correlation \\
\hline$(1.0,1.2)$ & 150 & $1.6 \pm 0.4$ & $0.29 \pm 0.14$ \\
$(1.2,1.6)$ & 155 & $2.4 \pm 0.5$ & $0.4 \pm 0.12$ \\
$(1.6,2.0)$ & 126 & $2.0 \pm 0.5$ & $0.3 \pm 0.15$ \\
\hline
\end{tabular}

$U / c$ were found only during the relatively high wind cases in which the wind did not turn markedly, so that the signal is lost in the noise. The noise is probably less due to instrumental and statistical error than to environmental variability and, of course, the inability of our simple model to describe a complex process. Thus the perplexing overshoot during case B7 does not appear to be experimental error. It is difficult to think of a model which would produce such an effect in the absence of additional environmental factors (swell, inhomogeneities, etc.). We do not believe that an improved model would yield radically different response times.

We believe the idealized case of a constant wind turning with an angular velocity $\Omega$ in a time and fetch unlimited situation to provide a useful asymptotic limit for examining various theoretical models of spectral evolution and to see in which combination the parameter $\Omega$ enters the dimensional analysis.

For this asymptotic case we have crudely estimated the total momentum flux to the waves, $\tau_{w}$ $=\dot{\mathbf{M}}_{w}$, which is perpendicular to the total wave momentum $\mathrm{M}_{w}$ and of magnitude $\Omega M_{u}$. We use the parameterization proposed by Hasselmann et al. (1976) for $E(f)$ and for the directional distribution $S(f, \theta)$ assume the validity of (6.3) with the mean spread $\theta_{2}(f)$ given by the equilibrium relations (4.6). In this case we find that $\Omega M_{w}$ is typically a few percent of the total momentum transfer out of the atmosphere, with the angle between $\mathbf{M}_{w}$ and $U$ depending on $\Omega U / g$. Thus, we conclude that our value for $b$ and the model (6.3) are consistent with earlier estimates (Hasselmann et al., 1973) of $C_{a d}$ for the total momentum flux to waves in simpler generation conditions.

An additional check on the time scales implied by Eq. (6.6) or Table 5 follows from the ideas of Mitsuyasu and Rikiishi (1978) concerning the growth of spectra in time-limited conditions. If in a homogeneous situation the wind suddenly shifts by $90^{\circ}$ the time it takes to turn the wavefield at the frequency $f$ into the new wind direction should roughly correspond to the time $T_{m}(f)$ it takes for the frequency $f$ to become the peak frequency in timelimited growth conditions. This analogy is only loose but the time scales should show some similarity.

If we take the results of Mitsuyasu and Rikiishi [1978, Eq. (7)] and use $u_{*}{ }^{2}=1.4 \times 10^{-3} U^{2}$, we obtain 


$$
\omega_{m} T_{m}=5.3 \times 10^{3}\left(c_{m} / U\right)^{0.75} .
$$

This response, observed in laboratory conditions for frequencies $f \geqslant 2 \mathrm{~Hz}$ is thus roughly 10 times faster than ours. For our frequency range we use the formula given by Mitsuyasu and Rikiishi (1978, Appendix)

where

$$
\tau=\frac{4 \pi}{1.34} \int_{0}^{\xi} \nu\left(\xi^{\prime}\right) d \xi^{\prime}
$$

$$
\nu=U f_{m} / g, \quad \tau=g T_{m} / U, \quad \xi=g F / U^{2}
$$

and $F$ is fetch. But now using the relation

$$
\nu=3.5 \xi^{-0.33}
$$

proposed by Hasselmann et al. (1973), we obtain

$$
\omega_{m} T_{m}=2.4 \times 10^{4}\left(c_{m} / U\right)
$$

which roughly agrees with our estimates for $b^{-1}$. [We point out that (6.13) depends sensitively on the exponent in (6.12).] We have been unable to detect any dependence of $b$ on $\left(c_{m} / U\right)$ as given by (6.13) or (6.10) for reasons explained above.

\section{Conclusions}

Our data show in generating conditions $U / c \geqslant 1$ that $s_{1}$ is best parameterized with the parameter $f / f_{m}$ with an additional dependence of the exponent in (4.6) on $U / c_{m}$. The scatter around the parametrical relation is larger than can be explained by sampling error and the origin of this scatter remains unexplained. Since our data base only contained four runs with values $U / c_{m}>1.5$, more experiments at small nondimensional fetch are desirable.

In applying the maximum likelihood fit to a large body of data we have found it to be a fast and stable procedure to assess data quality and improve data interpretation. We have found the ML results to be in close agreement with the results of an entirely different fitting procedure, indicating that unimodal and bimodal angular distributions can be fairly well estimated from pitch-and-roll information.

At 150 degrees of freedom the differences between the exact fits and those which fit only the first harmonic were statistically significant in $43 \%$ of all cases and in $28 \%$ of all steady wind cases.

As a first attempt to describe the response of the wavefield to a veering wind we have proposed the simple relation (6.3). The analysis yields an empirical time constant $T(\omega)$ such that

$$
\omega T(\omega)=b^{-1}=(4 \pm 1) \times 10^{4}
$$

for the response of the mean propagation angle to changes in wind direction. The model has obvious shortcomings, but we do not believe that a more sophisticated analysis will provide entirely differ- ent response times; thus (7.1) may serve as a guide for further work.

Acknowledgments. This work was supported by Deutsche Forschungsgemeinschaft by a grant to SFB 94 and through NATO (Grant 433). Numerical calculations mainly were done at the regional computer center RRZN in Hannover.

Thanks are expressed to R. B. Long and K. Hasselmann for providing us with the ML-fit routines and to all JONSWAP 73 participants for discussions and cooperation.

\section{APPENDIX A}

\section{The Meteorological Buoy}

The buoy has the shape of a circular cone, with diameter $2 L=2.08 \mathrm{~m}$ and height of $2.0 \mathrm{~m}$, and the cutoff frequency lies at $k L=1, f=0.5 \mathrm{~Hz}$. [see Hasse et al. (1978) for a more detailed description]. The wave-measuring equipment consists of a gyro, three accelerometers to measure the three accelerations $b_{1}{ }^{\prime}, b_{2}{ }^{\prime}, b_{3}{ }^{\prime}$ in a buoy-fixed frame, two inclination measurement devices $\left(\alpha_{1}{ }^{\prime}, \alpha_{2}{ }^{\prime}\right)$ to measure the inclinations of a buoy-fixed plane against the true (gyro-)vertical, a compass $\alpha_{3}$, and finally two pressure transducers to measure the submersion depth $d_{1}, d_{2}$ of the buoy.

All data are recorded at $20 \mathrm{~Hz}$; the $20 \mathrm{~Hz}$ data were then rotated to a data set $\left(b_{1}, b_{2}, b_{3}, \alpha_{1}, \alpha_{2}, \alpha_{3}\right.$; $d_{1}, d_{2}$ ) giving all data in the (east, north, vertical) system. The data were then filtered and decimated to a $2 \mathrm{~Hz}$ series. The buoy's response functions to tilt and heave can be obtained from the data. An independent calibration of the buoy would have been desirable, since it could have provided useful additional checks. Unfortunately, such an additional calibration was not available for the lack of a suitable facility. All components of the system must work to obtain a meaningful data set. If they do we obtain two sets of data which yield the same information within statistical error, since both the data set $\left(b_{1}, b_{2}, b_{3} ; d_{1}, d_{2}\right)$ and the data set $\left(\alpha_{1}, \alpha_{2}, b_{3} ; d_{1}, d_{2}\right)$ give the equivalent to pitch-and-roll information. Furthermore, the phases should and did behave according to linear wave theory, allowing for departures at buoy resonances, provided these occur approximately where they should, which is the case. With $d=1 / 2\left(d_{1}+d_{2}\right)$ and $\left\langle b_{i} b_{j}\right\rangle=C_{i j}+i Q_{i j} ; i, j$ $=1, \ldots, 3$ and $\left\langle\alpha_{i} \alpha_{j}\right\rangle=c_{i j}+i q_{i j}$ for $i, j=1,2$, $\left\langle\alpha_{i} b_{3}\right\rangle=c_{i 3}+i q_{i 3},\left\langle b_{3} d\right\rangle=C_{3 d}+i Q_{3 d}$ and $\langle d d\rangle$ $=C_{d d}$, the wave spectrum was obtained as

$$
E(f)=\omega^{-4} C_{33}-2 \omega^{-2} C_{3 d}+C_{d d}
$$

and the parameters $r_{i}^{(\nu)}, \delta_{i}^{(\nu)}$ were obtained from accelerations $(\nu=a)$ and inclinations $(\nu=i)$, as

$$
r_{1}^{(a)}=\left\{\frac{Q_{23}{ }^{2}+Q_{13}{ }^{2}}{C_{33}\left(C_{11}+C_{22}\right)}\right\}^{1 / 2},
$$




$$
\begin{aligned}
r_{2}^{(a)} & =\left\{\frac{\left(C_{11}-C_{22}\right)^{2}+4 C_{12}{ }^{2}}{\left(C_{11}+C_{22}\right)^{2}}\right\}^{1 / 2}, \\
\delta_{1}^{(a)} & =-\operatorname{artan}\left(\frac{Q_{23}}{Q_{13}}\right)+\pi / 2, \\
2 \delta_{2}^{(a)} & =-\operatorname{artan}\left(\frac{2 C_{12}}{C_{11}-C_{22}}\right)+\frac{\pi}{2} .
\end{aligned}
$$

Inclination values were obtained by the same formulas with $C_{i j}, Q_{i j}$ replaced by $c_{i j}, q_{i j}$.

As long as the buoy response is isotropic, not in resonance and linear, the directional analysis is insensitive to buoy response (except for a shift by $\pi$ when we are above the tilt resonance, which, indeed, had to be introduced for the inclination analysis; below resonance we should have replaced $Q_{i j}$ by $-q_{i j}$ ). In Fig. 10 we show typical results (run 251,0200 ) for the comparison of inclination (i) and acceleration (a) information.

There is some discrepancy between $r_{1}^{(a)}$ and $r_{1}^{(i)}$ particularly for $f<0.15 \mathrm{~Hz}$. We have not yet analyzed this point systematically - it is possible that inclinations are not so well correlated with vertical accelerations because they are more sensitive to the forces exerted by the mooring and the wind. We have found no case, just from a comparison of the (a) and (i) information, in which we would definitely say that the system was not measuring correctly. In the debugging phase of the program we found the results to be sensitive to errors in any instrument. Thus, for instance, an incorrect calibration formula for the angle $\alpha_{1}^{\prime}$ (wrong sign) was immediately seen in the results and the coherence $\left(b_{3}, d\right)$ which was 0.97 with the correct calibration dropped to 0.76 and the one-dimensional spectrum was also considerably distorted.

In Fig. 11 we show the transfer functions $T_{h}$ and $T_{p}{ }^{2}$ for heave and pitch, i.e.,

$$
\begin{aligned}
& T_{h}{ }^{2}=\omega^{-4} \frac{C_{33}}{E(f)}, \\
& T_{p}{ }^{2}=g^{2} \frac{C_{11}+C_{22}}{C_{33}} .
\end{aligned}
$$

The pitch resonance is so sharp that we must expect the buoy tilt to behave severely nonlinearly, whenever we have a wave field with considerable slope variance at resonance, but we have not yet looked at this point. For the data shown in Fig. 11 the variance $(\delta \alpha)^{2}$ integrated over the resonance yields $\delta \alpha \approx 5^{\circ}$. Since we believe that the inclination may

FIG. 10. A comparison of the directional information obtained from accelerations (light line) and inclinations measured with the meteorological buoy. The straight line in (c) and (d) shows the wind direction $\delta_{w}$. The frequency resolution is $\Delta f$ $=1 / 128 \mathrm{~Hz}$, corresponding to 30 degrees of freedom.
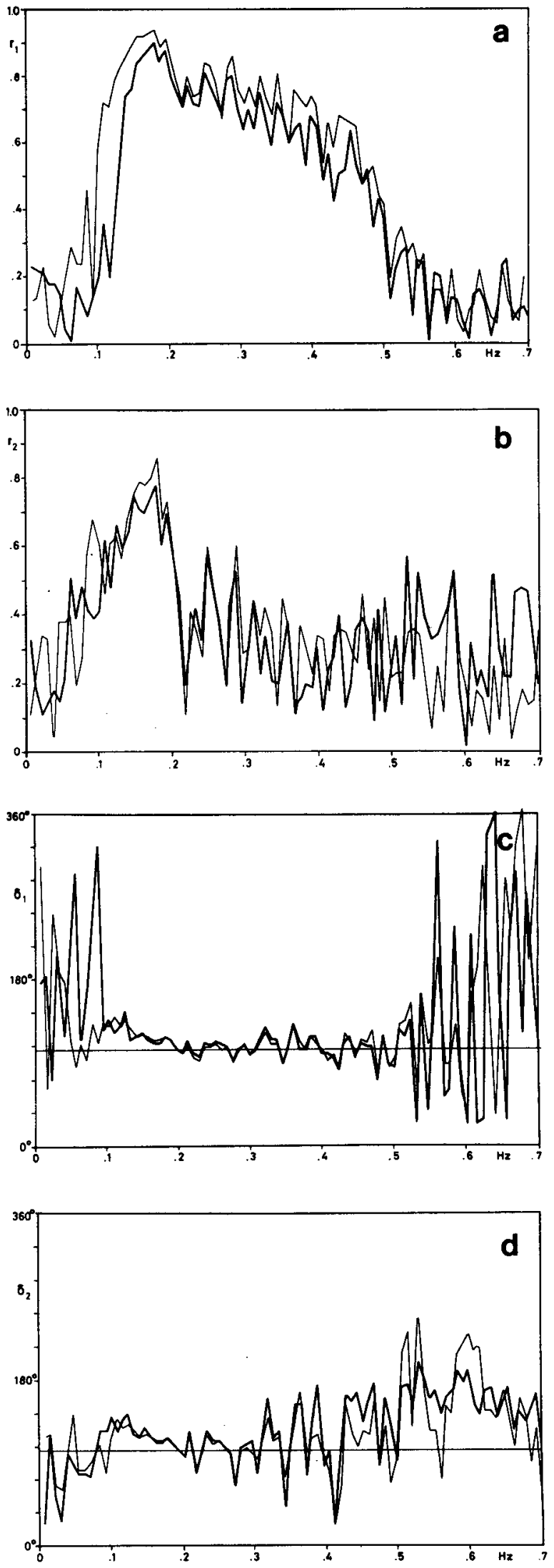


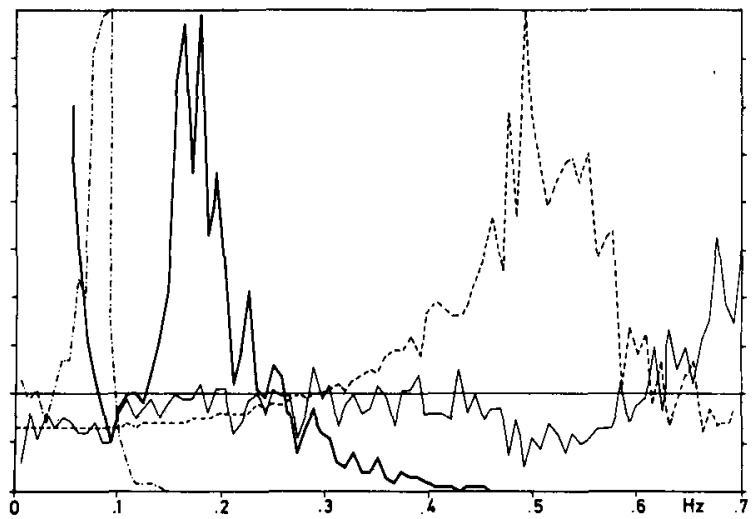

FIG. 11. One-dimensional wave spectrum $E(f)$ (heavy line) and transfer functions $T_{h}(f)(---), T_{p}{ }^{2}(f)(-\cdot)$ and $q^{2}(f)$ (solid line) for the same run and resolution as in Fig. 10. The straight line shows $q^{2}=1$. Scale runs from 0 to 5 for $q^{2}$, from 0 to $0.64 \mathrm{~m}^{2} \mathrm{~Hz}^{-1}$ for $E(f)$; from 0 to 221 for $T_{p}{ }^{2}$; and from 0.28 to 5.72 for $T_{h} . T_{h} \rightarrow 1$, for $f \rightarrow 0$.

be both more sensitive to other than wave forces and more likëly to be influenced by nonlinear response, we have in all but 29 runs used the acceleration data, which require only that the acceleration response be isotropic and linear for the final analysis. In the beginning 29 runs were analyzed with a faulty program, which gave incorrect values of $b_{1}$ and $b_{2}$ but was otherwise correct. Omission of these inclination runs (four of which fell into data set A) does not alter any of our conclusions, as is to be expected from the generally good agreement between the acceleration and inclination data, for which Fig. 10 is representative. For the acceleration runs the ratio

$$
q^{2}=\frac{\omega^{4} E(f)}{C_{11}+C_{22}}
$$

should yield a test for the dispersion relation for $k L \ll 1$. The results for $q^{2}$ shown in Fig. 11 are typical and we considered the value for $q^{2}$ to be in satisfactory agreement with the dispersion relation.

After analyzing all runs we averaged $q$ over all runs to obtain the results shown in Fig. 12. We are not worried about the deviations for $f \geqslant 0.25$ $\mathrm{Hz}$, since $k L \geqslant 0.25$ here and the values of $q$ already may well be influenced by the geometry of the buoy. We have no explanation for the departure from the dispersion curve for the poor frequencies $0.1<f$ $<0.2 \mathrm{~Hz}$, while again for lower frequencies the wave signal is so weak that many explanations are plausible. In the poor region it is hard to see how some $10-20 \%$ of the acceleration signals could be due to noise (mooring, instrumental noise, nonlinear buoy response, accelerations due to rotation rather than translation of the buoy), but it is even harder to believe that the wave field should show such a strong departure from linear theory. For now the problem remains unresolved, but we plan to look into this matter - we also plan to test whether the departures for $k L \geqslant 0.25$ are really explained by buoy properties.

We have postponed this analysis, because as stated above, away from resonance as long as the response remains linear and isotropic, the directional information is not influenced. The poor frequency region causès more concern, since noise of any sort will to some degree influence the directional information, except for $\delta_{1}$. We do not believe this results in a serious error, but some reserve is expressed regarding the poor frequency region; nevertheless, these frequency bands were eventually treated on an equal footing in the overall analysis.

\section{APPENDIX B}

\section{The 2M Fit}

We first rotate to $\tilde{\delta}_{1}=0$, so that we now have $r_{1}, r_{2}, \tilde{\delta}_{1}=0, \tilde{\delta}_{2}=\delta_{2}-\delta_{1}$. We then introduce $\left(q_{1}, q_{2}\right)$ as the $\left(r_{1}, r_{2}\right)$ values of $M\left(\theta, p_{1}, \beta_{1}\right)$ and $\left(Q_{1}, Q_{2}\right)$ as those of $M\left(\theta, p_{2}, \beta_{2}\right)$ in Eq. (5.2). Thus

$$
q_{1}=\frac{p_{1}}{p_{1}+1}, \quad q_{2}=\frac{p_{1}\left|p_{1}-1\right|}{\left(p_{1}+1\right)\left(p_{1}+2\right)},
$$

and $Q_{1}, Q_{2}$ are obtained from $p_{2}$.

Thus, we obtain

$$
\begin{aligned}
& r_{1}=\lambda q_{1} \cos \beta_{1}+(1-\lambda) Q_{1} \cos \beta_{2}, \\
& 0=\lambda q_{1} \sin \beta_{1}+(1-\lambda) Q_{1} \sin \beta_{2} \text {, } \\
& \epsilon_{3}=r_{2} \cos 2 \tilde{\delta}_{2}-\lambda q_{2} \cos 2 \hat{\beta}_{1} \\
& -(1-\lambda) Q_{2} \cos 2 \beta_{2}=0, \\
& \epsilon_{4}=r_{2} \sin 2 \tilde{\delta}_{2}-\lambda q_{2} \sin 2 \beta_{1} \\
& -(1-\lambda) Q_{2} \sin 2 \beta_{2}=0 .
\end{aligned}
$$

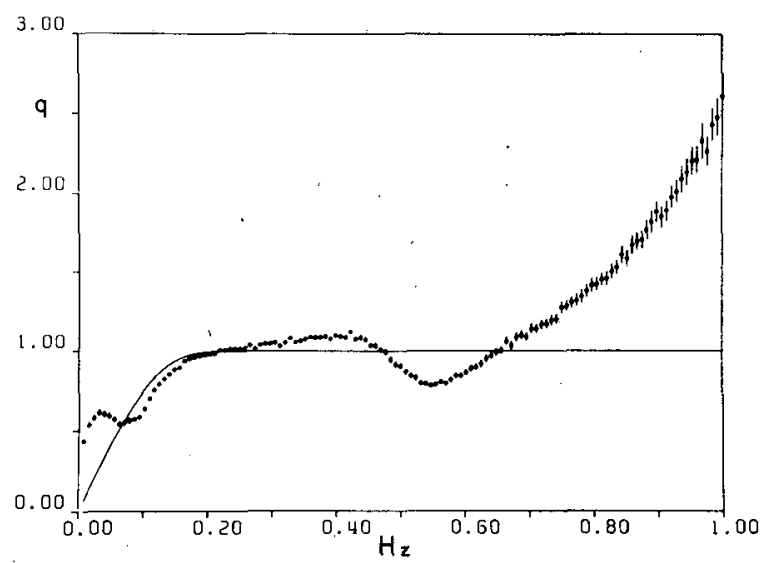

FIG. 12. Mean transfer function $q$ averaged over all 137 acceleration runs, error bars showing standard deviation of the mean. The line shows the dispersion relation $q=\omega^{2}(k g)^{-1}$ for the mean water depth of $18 \mathrm{~m}$ at station 8 . The deviations for frequencies $0.08<f<0.20 \mathrm{~Hz}$ are not satisfactorily explained. 
After solving (B2) and (B3) for $\beta_{1}$ and $\beta_{2}$, i.e.,

$$
\begin{aligned}
& \cos \beta_{1}=\frac{1+{\rho_{1}}^{2}-{\rho_{2}}^{2}}{2 \rho_{1}}, \\
& \cos \beta_{2}=\frac{1+{\rho_{2}}^{2}-{\rho_{1}}^{2}}{2 \rho_{2}},
\end{aligned}
$$

where

$$
\rho_{1}=\frac{\lambda q_{1}}{r_{1}}, \quad \rho_{2}=(1-\lambda) Q_{1} / r_{1},
$$

and $\sin \beta_{1} \sin \beta_{2} \leqslant 0$, we can insert these results into (B4) and (B5), which thus yield a fairly simple set of equations in the parameters $\left(\lambda, p_{1}, p_{2}\right)$; in the numerics we actually worked with $\left(\lambda, q_{1}, Q_{1}\right)$. The simple parametrical relations between $\left(q_{1}, q_{2}\right)$ and $\left(Q_{1}, Q_{2}\right)$ are the main advantage of $1 \mathrm{M}$ functions as structure functions - otherwise any one-parameter unit-integral-structure centered on an angle (e.g., a box or a triangle) could just as well be used in Eqs. (B2)-(B5).

Our method of solution was simply to minimize

$$
\begin{gathered}
\epsilon^{2}=w_{3} \epsilon_{3}^{2}+w_{4} \epsilon_{4}^{2}+w_{5} \epsilon_{5}^{2}, \\
\epsilon_{5}=p_{1}-p_{2}=\Delta p,
\end{gathered}
$$

and the weights $w_{i}$ had to be varied to detect and escape from undesired side minima of $\epsilon^{2}$. A solution was accepted as perfectly satisfactory if $\epsilon_{3}{ }^{2}+\epsilon_{4}{ }^{2}$ $<4 \times 10^{-6}$ and $\Delta^{2} p<10^{-4}$. (It can be shown that $\Delta p \equiv 0$ is a too restrictive condition to be generally imposed.) If only the condition on $\Delta p$ was not satisfied, the solution with the minimum $\Delta^{2} p$ was taken. If no solution satisfying $\epsilon_{3}{ }^{2}+\epsilon_{4}{ }^{2}<4 \times 10^{-6}$ was found, the case was classified as a $2 \mathrm{M}$-failure.

\section{APPENDIX C}

\section{Error Analysis}

The following relations hold for the usual reduction of pitch-and-roll data as used for the data obtained at station 10. Minor modifications resulting from Eqs. (A2)-(A5) (Long, 1979), as used for the data obtained with the meteorological buoy, were included. We turn the directional distribution $S(\theta)$ $=M(\theta, s, \delta)$ to $\delta=0$ and can estimate the parameters from simplified expressions if we neglect terms $\mathrm{O}(1 / \nu)^{2}$ in the associated error matrix. The results are

$$
\begin{aligned}
r_{1} & =\frac{Q_{13}}{C_{33}} \geqslant 0, \\
r_{2} & =\frac{C_{11}-C_{22}}{C_{33}}, \\
\delta_{1} & =\operatorname{artan}\left(Q_{23} / Q_{13}\right), \\
2 \delta_{2} & =\operatorname{artan}\left(\frac{2 C_{12}}{C_{11}-C_{22}}\right),
\end{aligned}
$$

where the inequality $(\mathrm{C} 1)$ is satisfied if we rotate our coordinate system accordingly. The covariance matrix of the estimators $\hat{C}_{i j}$ and $\hat{Q}_{i j}$ is known (see, e.g., Müller et al., 1978) which yields, writing, e.g., $\hat{r}_{1}=r_{1}+r_{1}^{\prime}$ for the mean and error of the estimators,

$$
\begin{aligned}
& \nu\left\langle r_{1}{ }^{\prime} r_{1}{ }^{\prime}\right\rangle=1 / 2\left(1+r_{2}\right)-r_{1}{ }^{2}, \\
& \nu\left\langle r_{2}{ }^{\prime} r_{2}{ }^{\prime}\right\rangle=2\left[1 / 2\left(1+3 r_{2}{ }^{2}\right)-2 r_{2} r_{1}{ }^{2}\right], \\
& \nu\left\langle r_{1}{ }^{\prime} r_{2}{ }^{\prime}\right\rangle=r_{1}\left(1+r_{2}\right)-2 r_{1}{ }^{3}, \\
& \nu\left\langle\delta_{1}{ }^{\prime} \delta_{1}{ }^{\prime}\right\rangle=1 / 2\left(1-r_{2}\right) r_{1}{ }^{-2}, \\
& \nu\left\langle\delta_{2}{ }^{\prime} \delta_{2}{ }^{\prime}\right\rangle=1 / 4\left(1-r_{2}{ }^{2}\right) r_{2}{ }^{-2}, \\
& \nu\left\langle\delta_{1}{ }^{\prime} \delta_{2}{ }^{\prime}\right\rangle=1 / 2\left(1-r_{2}\right) r_{2}{ }^{-1} .
\end{aligned}
$$

All other matrix elements vanish at $O(1 / \nu)$. These estimates are obtained under the approximation that $\hat{r}_{1}, \hat{r}_{2}, \hat{\delta}_{1}$ and $\hat{\delta}_{2}$ have a joint normal distribution, which is poor for $\left|r_{1}-1\right| \ll 1 / \nu$ and $r_{1} \ll 1 / \nu$. We have, similar to the usual treatment of coherences, therefore transformed to $\rho_{1}$ and $\rho_{2}$, where

so that

$$
\rho_{i}=\tanh ^{-1}\left(r_{i}\right), \quad i=1,2,
$$

$$
\rho_{i}^{\prime}=\frac{d \rho_{i}}{d r_{i}} r_{i}^{\prime}
$$

Having thus determined the error matrix $V_{i j}$ of our estimators $\hat{p}_{i}=\left(\hat{\rho}_{1}, \hat{\rho}_{2}, \hat{\delta}_{1}, \hat{\delta}_{2}\right)$ the value of $\chi^{2}$ is determined from

$$
\chi^{2}=\epsilon_{i} V_{i j}{ }^{-1} \epsilon_{j},
$$

where $\epsilon_{i}=p_{i}-P_{i}$, and $p_{i}$ are the model and $P_{i}$ the measured parameters. In this treatment the bias of $\hat{r}_{1}$ and $\hat{r}_{2}$ at low values of $r_{1}$ and $r_{2}$ is not included.

The use of the variables $\rho_{1}, \rho_{2}, \delta_{1}, \delta_{2}$ has some obvious disadvantages regarding their statistical properties, but they are simple to use because of the simple covariance matrix $V_{i j}$. A more rigorous treatment starting directly from the $C_{i j}$ and $Q_{i j}$ (Long, 1979) would probably not have produced results differing much from ours. Note that $\operatorname{det} V \rightarrow 0$ as $(1 / s)^{2}$ for $1 / s \rightarrow 0$, so that a model with a high $s$ parameter will produce high values of $\chi^{2}$ for comparatively low values of $\epsilon^{2}$.

\section{REFERENCES}

Brümmer, B., D. Heinricy, L. Krügermeyer and D. Prümm, 1974: The large-scale weather features over the North Sea during the JONSWAP II experiment. Berichte des Instituts für Radiometeorologie und Maritime Meteorologie, No. 24. [Available from Meteor. Inst., Universität Hamburg].

Cartwright, D. E., 1963: The use of directional spectra in studying the output of the wave-recorder on a moving ship. Ocean Wave Spectra, Prentice Hall, 203-218.

, and N. D. Smith, 1964: Buoy techniques for obtaining directional wave spectra. Buoy Technology, Mar. Tech. Soc., 112-121.

Cote, L. J., J. O. Davis, W. Marks, R. J. McGough, E. Mehr, 
W. J. Pierson, Jr., J. F. Ropek, G. Stephenson and R. C. Vetter, 1960: The directional spectrum of a wind-generated sea as determined from data obtained by the stereo wave observation project. Meteor. Pap. NYU, College of Engineering, 2, No. 6, $88 \mathrm{pp}$.

Ewing, J. A., 1969: Some measurements of the directional wave spectrum. J. Mar. Res., 27, 163-171.

Forristall, G. Z., E. G. Ward, V. J. Cardone and L. E. Borgmann, 1978: The directional spectra and kinematics of surface gravity waves in Tropical Storm Delia. J. Phys. Oceanogr., 8, 888-909.

Günther, H., W. Rosenthal and K. Richter, 1979: Application of the parametrical wave prediction model to rapidly varying wind fields during JONSWAP 1973. J. Geophys. Res., $84,4855-4864$.

Hasse, L., M. Grünewald, J. Wucknitz, M. Dunckel and D. Schriever, 1978: Profile derived turbulent fluxes in the surface layer under disturbed and undisturbed conditions during GATE. Meteor. Forsch.-Ergebnisse, B13, 24-40.

Hasselmann, K., D. B. Ross, P. Müller and W. Sell, 1976: A parametric wave prediction model. J. Phys. Oceanogr., 6, 200-228.

- , T. P. Barnett, E. Bouws, H. Carlson, D. E. Cartwright, K. Enke, J. A. Ewing, H. Gienapp, D. E. Hasselmann, P. Kruseman, A. Meerburg, P. Müller, D. J. Olbers, K. Richter, W. Sell and H. Walden, 1973: Measurements of windwave growth and swell decay during the Joint North
Sea wave project (JONSWAP). Dtsch. Hydrogr. Z., A8(Suppl.), No. 12.

Long, R. B., 1980: The statistical evaluation of directional spectrum estimates derived from pitch/roll buoy data. J. Phys. Oceanogr., 10, 944-952.

extracting directional spectra from multicomponent wave data. J. Phys. Oceanogr., 9, 373-381.

Longuet-Higgins, M. S., D. E. Cartwright and N. D. Smith, 1963: Observations of the directional spectrum of sea waves using the motions of a floating buoy. Ocean Wave Spectra, Prentice Hall, 111-136.

Mitsuyasu, H., and K. Rikiishi, 1978: The growth of duration limited wind waves. J. Fluid Mech., 85, 705-730.

_ , F. Tasai, T. Suhara, S. Mizuno, M. Ohkuso, T. Honda and K. Rikiishi, 1975: Observations of the directional spectrum of ocean waves using a cloverleaf buoy. J. Phys. Oceanogr., $5,750-760$.

Müller, P., D. J. Olbers and J. Willebrand, 1978: The IWEX spectrum. J. Geophys. Res., 83, 479-499.

Regier, L. A., and R. E. Davis, 1977: Observation of the power and directional spectrum of ocean surface waves. J. Mar. Res., 35, 434-451.

Tyler, G. L., C. C. Teague, R. H. Stewart, A. M. Peterson, W. H. Munk and J. W. Joy, 1974: Wave directional spectra from synthetic aperture observations of radio scatter. Deep-Sea Res., 21, 989-1016. 